Mannheimer Mathematische Manuskripte $185 \% 94$

\title{
YANG-MILLS AND DIRAC FIELDS IN A BAG, CONSTRAINTS AND REDUCT́TION
}

\author{
Jędrzej Śniatycki ${ }^{*}$, Günter Schwarz ${ }^{\star}$ and Larry Bates ${ }^{\dagger}$.<smiles>[131In]</smiles>

\begin{abstract}
\end{abstract}
The structure of the constraint set in the Yang-Mills-Dirac theory in a contractible bounded domain is analysed under the bag boundary conditions. The gauge symmetry group is identified, and it is proved that its action on the phase space is proper and admits slices.

The reduced phase space is shown to be the union of symplectic manifolds, each of which corresponds to a definite mode of symmetry breaking.

Department of Mathematics and Statistics, University of Calgary, Calgary, Alberta. Canada. Research partially supported by NSERC Research Grant SAP0008091.

* Department of Mathematics and Computer Science, University of Mannheim, 6800 Mannheim, Germany. Research partially supported by DFG Grant Schw. 485/2-1.

$\dagger$ Department of Mathematics and Statistics, University of Calgary, Calgary, Alberta, Canada. Research partially supported by NSERC Research Grant OGPO0424 16. 


\section{Introduction.}

In a previous paper we have proved the existence and uniqueness theorems for minimally interacting Yang-Mills and Dirac fields in a bounded contractible domain $\mathbf{M} C$ $\mathbb{R}^{3},[1]$. The aim of this paper is to study the structure of the space of solutions.

Our results were obtained for Cauchy data $A \in H^{2}(M), \quad E \in H^{l}(M)$, and $\Psi \in \mathrm{H}^{2}(\mathrm{M})$, where and $\mathrm{H}^{\mathrm{k}}(\mathrm{M})$ is the Sobolev space of fields on $M$ which are square integrable together with their derivatives up to the order $k$, satisfying the boundary conditions

$$
\begin{gathered}
n \mathrm{E}=0, \quad t \mathrm{~B}=0,\left.\quad i n_{\mathrm{j}} \gamma \dot{\gamma}\right|_{\partial \mathrm{M}}=\left.\Psi\right|_{\partial \mathrm{M}} \\
n \mathrm{~A}=0,\left.i n_{\mathrm{j}} \dot{\gamma}\left\{\gamma\left(\gamma \mathrm{\gamma} \partial_{\mathrm{k}}+i m\right) \Psi\right\}\right|_{\partial \mathrm{M}}=\left.\gamma\left(\gamma^{k} \partial_{\mathrm{k}}+i m\right) \Psi\right|_{\partial \mathrm{M}} .
\end{gathered}
$$

Here we use the notation established in [1]. In particular, $n \mathrm{E}$ denotes the normal component of the "electric" part, $t \mathrm{~B}$ the tangential component of the "magnetic" part of the field strength on the boundary $\partial \mathrm{M}$ of $\mathrm{M}$. Thus, the extended phase space of the theory under consideration is

$$
P=\left\{(A, E, \Psi) \in H^{2}(M) \times H^{1}(M) \times H^{2}(M) \mid \text { satisfying }-(1.1 a, b)\right\}
$$

The variational principle underlying the theory gives rise to a (weak) symplectic structure on $P$. Let $\theta$ be a 1 -form on $P$ such that, for everry $p=(A, E, \Psi) \in P$ and $a \frac{\delta}{\delta A}$ $+\mathrm{e}^{\delta} \frac{\delta \mathrm{E}}{\delta} \psi_{\delta \Psi}^{\delta} \in \mathrm{T}_{\mathrm{p}} \mathrm{P}$

$$
\left\langle\theta(\mathrm{A}, \mathrm{E}, \Psi) \mid \mathrm{a}_{\delta \mathrm{A}}^{\delta}+\mathrm{e}_{\delta \mathrm{E}}^{\delta}+\psi_{\delta \Psi}^{\delta}\right\rangle=\int_{\mathrm{M}}\left(\mathrm{E} \cdot \mathrm{a}+\psi^{\dagger} \psi\right) \mathrm{d}_{3} \mathrm{x},
$$

The symplectic form $\omega$ of $\mathbf{P}$ is the exterior differential of $\theta$,

$$
\omega=\mathrm{d} \theta \text {. }
$$

Let $G$ be the structure group of the theory, presented as a matrix group, and $g$ be the Lie algebra of $G$. We assume that $G$ is compact, and that $g$ admits an ad-invariant metric. The group GS(P) of gauge symmetries consists of maps $\phi: \mathrm{M} \rightarrow \mathrm{G}$ such that their action on the variables (A,E, $\Psi$ ), given by 


$$
\mathrm{A} \mapsto \phi \mathrm{A} \phi^{-1}+\phi g r a d \phi^{-1}, \mathrm{E} \mapsto \phi \mathrm{E} \phi^{-1}, \Psi=\phi \Psi,
$$

leaves the extended phase space $P$ invariant. The infinitesimal action of an element $\xi$ of the Lie algebra $g s(\mathrm{P})$ of $G S(\mathrm{P})$ is given by

$$
\mathrm{A} \mapsto \mathbf{A}-\mathrm{D}_{\mathrm{A}} \boldsymbol{\xi}, \quad \mathrm{E} \mapsto \mathrm{E}-[\mathrm{E}, \xi], \quad \Psi=\Psi+\xi \Psi,
$$

where

$$
\mathrm{D}_{\mathrm{A}} \xi=\operatorname{grad} \xi+[\mathrm{A}, \xi]
$$

is the covariant derivative of $\xi$ with respect to the connection defined by $A$. It gives rise to a vector field $\xi_{\mathrm{P}}$ on $\mathrm{P}$ such that

$$
\xi_{\mathrm{P}}(\mathrm{A}, \mathrm{E}, \Psi)=-\left(\mathrm{D}_{\mathrm{A}} \xi\right) \frac{\delta}{\delta \mathrm{A}}-[\mathrm{E}, \xi] \frac{\delta}{\delta \mathrm{E}}+\xi \Psi \frac{\delta}{\delta \Psi}
$$

The action of $G S(P)$ preserves the 1 -form $\theta$. Hence, it is Hamiltonian with the equivariant momentum map $\mathrm{J}: \mathrm{P} \rightarrow g s(\mathrm{P})^{*}$ such that

$$
\langle\mathrm{J}(\mathrm{A}, \mathrm{E}, \Psi) \mid \xi\rangle=\left\langle\theta \mid \xi_{\mathrm{P}}(\mathrm{A}, \mathrm{E}, \Psi)\right\rangle=\int_{M}\left\{-\mathrm{E} \cdot \mathrm{D}_{\mathrm{A}} \xi+\Psi^{\dagger} \xi \Psi\right\} \mathrm{d}_{3} \mathrm{x}
$$

Here $g s(\mathrm{P}){ }^{*}$ denotes the $\mathrm{L}^{2}$ dual of $g s(\mathrm{P})$, that is the space of square integrable maps from $M$ to the dual $\mathfrak{g}^{*}$ of the Lie algebra $\mathfrak{g}$ of the structure group $G$. For each $\xi \in$ $g s(\mathrm{P})$, the function $\mathrm{J}_{\xi}: \mathrm{P} \rightarrow \mathbb{R}$ given by

$$
\mathrm{J}_{\xi}(\mathrm{A}, \mathrm{E}, \Psi)=\langle\mathrm{J}(\mathrm{A}, \mathrm{E}, \Psi) \mid \xi\rangle
$$

is called the momentum associated to $\xi$. The vector field $\xi_{\mathbf{P}}$ is the Hamiltonian vector field of $J_{\xi}$, i.e.

$$
\left.\xi_{\mathrm{P}}\right\lrcorner \omega=\mathrm{dJ}_{\xi}
$$

Integrating by parts on the right hand side of Eq. (1.9), and taking into account the boundary condition $n \mathrm{E}=0$, we obtain

For every $\xi \in g s(\mathbf{P})$,

$$
\langle\mathrm{J}(\mathrm{A}, \mathrm{E}, \Psi) \mid \xi\rangle=\int_{\mathrm{M}}\left\{(\operatorname{div} \mathrm{E}+[\mathrm{A} ; \mathrm{E}]) \xi+\psi^{\dagger} \xi \Psi\right\} \mathrm{d}_{3} \mathrm{x} .
$$

$$
\Psi^{\dagger} \xi \Psi=-j \cdot \xi,
$$

where $j$ is the source term in the Yang-Mills-Dirac theory. Hence, the constraint equation of the theory 


$$
\operatorname{div} \mathrm{E}+[\mathrm{A} ; \mathrm{E}]=j,
$$

is equivalent to the vanishing of the momentum map $\mathrm{J}$.

The presentation of the constraint set as the zero level $\mathrm{J}^{-1}(0)$ of the momentum map $\mathrm{J}$, enables one to study its structure in terms of the action of the group of gauge symmetries. It was first done by Arms [2], who discussed the structure of the constraint set for pure Yang-Mills fields in compact spaces (no boundary) in general terms, without specifying the topology of the function spaces under consideration. The structure of the zero level of the momentum map, corresponding to a Hamiltonian action of a Hilbert-Lie group on a Hilbert manifold was studied; under additional technical assumptions, by Arms, Marsden and Moncrief, [3]. Special cases were considered by Mitter and Vialet [4], Atiyah and Bott [5], Kondracki and Rogulski [6] and Huebschmann $[7,8]$.

Functional analytic, assumptions made in this paper are consequences of the results of, [1]. They fail to satisfy two basic assumptions made in [3]: (i) neither the differential of $\mathrm{J}$ nor its adjoint are elliptic, (ii) the extended phase space $\mathbf{P}$ is not invariant under the interchange of A and E. Hence, we cannot use the results of Arms, Marsden and Moncrief, [3]. Instead, we follow the main idea of their paper, and prove the necessary intermediate steps. In particular, we prove the properness of the action of $G S(\mathbf{P})$ and of the existence of slices for this action. From this we show that the reduced phase space is the union of symplectic manifolds labelled by the conjugacy classes of compact subalgebras of,$g s(\mathrm{P})$. Each of these symplectic manifolds consists of the fields (A,E, $\Psi)$ with a definite mode of symmetry breaking.

In the finite dimensional case the partition of the reduced phase space into symplectic manifolds can be refined using conjugacy classes of compact subgroups of $G S(\mathrm{P})$ rather than compact subalgebras. In this case one obtains a stratification, with strata which can described algebraically in terms of the Poisson algebra, c.f. $[9 ; 10]$. 
Similar results for central Yang-Mills connections on surfaces has been obtained in [8]. An adaptation of their approach to our phase space will be studied elsewhere.

The paper is organized as follows. In Section 2 we discuss, in a proper functional analytic framework, the gauge symmetry group and its action. The structure of the zero level of the momentum map is analysed in Section 3. A stratification of the reduced phase space is studied in Section 4. Section 5 contains discussion of symmetry breaking corresponding to each stratum. The almost complex structure in the $\mathrm{L}^{2}$ completion of $\mathrm{P}$ is discussed in Appendix A. The properness of the action of $G S(P)$ is proved in Appendix B. The slice theorem is proved in Appendix C.

\section{Gauge Symmetries and the Momentum Map.}

The requirement that (1.6) gives an action of $\xi \in g s(\mathrm{P})$ in the space $\mathbf{P}$, defined by (1.2), implies that $\operatorname{grad} \xi \in \mathrm{H}^{2}(\mathrm{M})$. Since $M$ is bounded, it follows that $\xi \in \mathrm{H}^{3}(\mathrm{M})$. Moreover, the action of $\xi$ has to preserve the boundary conditions. The conditions (1.1a) are the usual bag boundary, conditions and are gauge invariant. The conditions (1.1b) are satisfied if and only if $n \cdot \operatorname{grad} \xi=0$. Hence,

$$
\operatorname{gs}(\mathrm{P})=\left\{\xi: \mathrm{M} \rightarrow \mathfrak{g} \mid \xi \in \mathrm{H}^{3}(\mathrm{M}) \text { and } n \cdot \operatorname{grad} \xi=0\right\} .
$$

The $\mathrm{L}^{2}$ dual $g s(\mathbf{P})^{*}$ of $g s(\mathrm{P})$, considered here, is the space of square integrable maps from $M$ to the dual $\mathfrak{g}^{*}$ of $\mathfrak{g}$, that is

$$
g s(\mathrm{P})^{*}=\left\{v: \mathrm{M} \rightarrow \mathfrak{g}^{*} \mid v \in \mathrm{L}^{2}(\mathrm{M})\right\}
$$

The evaluation of $v \in g s(\mathrm{P})^{*}$ on $\xi \in g s(\mathrm{P})$ is given by pointwise evaluation and integration

$$
\langle v \mid \xi\rangle=\int_{\mathrm{M}} v \cdot \xi \mathrm{d}_{3} \mathrm{x}
$$

The momentum map $\mathrm{J}$ defined in Eq. (1.9) is a continuous map from $\mathbf{P}$ to $g s(\mathbf{P})^{*}$. $G S(\mathrm{P})$ has a manifold structure with the tangent bundle space spanned by $g s(\mathrm{P})$. The presentation of the structure group $G$ as a matrix group, and boundedness. 
- of $\mathrm{M}$, enable us to present $G S(\mathrm{P})$ as a group of maps $\phi$ from $\mathrm{M}$ to $\mathrm{G}$ of Sobolev class $\mathrm{H}^{3}(\mathrm{M})$. Moreover, the boundary conditions (1.1) require that $n \cdot \operatorname{grad} \phi=0$. Hence,

$$
G S(\mathbf{P})=\left\{\phi: \mathrm{M} \rightarrow \mathrm{G} \mid \phi \in \mathrm{H}^{3}(\mathrm{M}) \text { and } n \cdot \operatorname{grad} \phi=0\right\} .
$$

Since $M$ is contractible and $G$ is connected, $G S(P)$ is connected. However, it need not be simply connected.

\section{PROPOSITION 2.1.}

The exponential mapping exp $: g s(\mathbf{P}) \rightarrow G S(\mathbf{P})$ is a diffeomorphism of a neighbourhood of $0 \in g s(\mathbf{P})$ onto a neighbourhood of the identity in $G S(\mathbf{P})$.

Proof. Let $U$ be a neighbourhood of $0 \in \mathfrak{g}$ and $\mathrm{V}$ a neighbourhood of the identity $e \in \mathrm{G}$, such that the exponential mapping $\exp : \mathfrak{g} \rightarrow \mathrm{G}$ is a diffeomorphism of $\mathrm{U}$ onto $\mathrm{V}$, and let $\ln : \mathrm{V} \rightarrow \mathrm{U}$ be the inverse of this diffeomorphism. Since, by the Sobolev embedding theorem, each $\phi \in G S(\mathrm{P})$ is a continuous map from $\mathrm{M}$ to $\mathrm{G}$, the sets.

$$
\mathbf{V}=\left\{\phi \in G S(\mathbf{P}) \mid \text { range } \phi \subseteq \mathrm{V}_{\mid}\right\} \text {. }
$$

is open in $G S(\mathrm{P})$. Similarly, the set

$$
\mathrm{U}=\{\xi \in g s(\mathrm{P}) \mid \text { range } \xi \subseteq \mathrm{U}\}
$$

is open in $g s(\mathrm{P})$. For every $\phi \in \mathrm{V}, \ln \circ \phi$ is in $g s(\mathrm{P})$, and its range is in U. Hence, lno $\phi$ $\in \mathrm{U}$. Let $\exp : g s(\mathrm{P}) \rightarrow G S(\mathrm{P})$ denote the exponential for the gauge algebra. For every $\xi \in g s(\mathrm{P}), \exp (\xi)=\exp \xi$. Hence, for every $\phi \in \mathrm{V}, \exp (\ln \circ \phi)=\exp \ln \circ \phi=\phi$, which implies that $\exp (\mathrm{U})=\mathrm{V}$.

The main property of the action of $G S(\mathbf{P})$ in $\mathbf{P}$ used in this paper is its properness. 
THEOREM 1. The action of $G S(P)$ in $\mathrm{P}$ is proper.

That is, for every sequence $p_{n}$ converging to $q$ in $P$ and every sequence $\phi_{n}$ in $G S(\mathrm{P})$ such that $\phi_{\mathrm{n}} \mathrm{p}_{\mathrm{n}}$ converges to $\mathrm{p}$, the sequence $\phi_{\mathrm{n}}$ has a convergent subsequence with limit $\phi$, and $\phi \mathrm{q}=\mathrm{p}$.

ProOF is given in Appendix B.

For each $\mathrm{p} \in \mathbf{P}$, we denote by $O_{\mathrm{p}}$ the orbit of $G S(\mathrm{P})$ through $\mathrm{p}$,

$$
O_{\mathrm{p}}=\{\phi \mathrm{p} \mid \phi \in G S(\mathrm{P})\}
$$

All orbits $O_{\mathrm{p}}$ of $G S(\mathbf{P})$ are closed since, if $\phi_{\mathrm{n}} \mathrm{p}$ is a convergent sequence of points in $O_{\mathrm{p}}$ with limit $\mathrm{q}$, then the sequence $\phi_{\mathrm{n}}$ has a convergent subsequence with limit $\phi$ and $\mathrm{q}$ $=\phi \mathrm{p}$, which implies that $\mathrm{q} \in O_{\mathrm{p}}$.

For every subspace $V$ of $T_{p} P$, we denote by $V^{\omega}$ the symplectic annihilator of $V$, that is

$$
\mathbf{V}^{\omega}=\left\{\mathbf{w} \in \mathrm{T}_{\mathrm{p}} \mathbf{P}|\omega(\mathbf{v}, \mathbf{w})=0 \quad \forall \quad \mathbf{v}| \in \mathbf{V}\right\}
$$

Note that that $\mathrm{V}^{\omega}$ is closed, and if $\mathrm{V}^{\mathrm{i}}$ closed, then $\left(\mathrm{V}^{\omega}\right)^{\omega}=\mathrm{V}$.

\section{Proposition 2.2.}

For each $\mathbf{p} \in \mathbf{P}$,

$$
\mathrm{T}_{\mathbf{p}} O_{\mathbf{p}}=\left(\text { ker } \mathrm{d}_{\mathbf{p}}\right)^{\omega}
$$

ProOf. If $\xi_{\mathrm{P}}$ is the Hamiltonian vector field of $\mathrm{J}_{\xi}$, c.f. Eq. (1.11), then for every $\mathbf{v}$ $\epsilon T_{p} P$

$$
\omega\left(\xi_{\mathbf{p}}(\mathrm{p}), \mathrm{v}\right)=\left\langle\mathrm{dJ}_{\mathbf{p}}(\mathrm{v}) \mid \xi\right\rangle
$$

Since $\mathrm{T}_{\mathbf{p}} O_{\mathbf{p}}=\left\{\xi_{\mathbf{p}}(\mathrm{p}) \mid \xi \in g s(\mathrm{P})\right\}$ it follows that $\mathbf{v} \in\left(\mathrm{T}_{\mathbf{p}} O_{\mathbf{p}}\right)^{\omega}$ if and only if 
$\mathbf{v} \in \operatorname{ker} \mathrm{dJ}_{\mathbf{p}}$. Hence, $\left(\mathrm{T}_{\mathbf{p}} O_{\mathbf{p}}\right)^{\omega}=\operatorname{ker} \mathrm{dJ}_{\mathbf{p}}$, and therefore $\mathrm{T}_{\mathbf{p}} O_{\mathbf{p}}=\left(k e r \mathrm{dJ}_{\mathbf{p}}\right)^{\omega}$, since ker $\mathrm{dJ}_{\mathbf{p}}$ is closed.

Q.E.D.

\section{PROPOSITION 2.3}

For every $\mathbf{p} \in \mathbf{P}$, range $\mathrm{dJ}_{\mathbf{p}}$ is a closed subspace of $g s(\mathbf{P})^{*}$ with finite codimension.

Proof. For $\mathbf{p}=(\mathbf{A}, \mathrm{E}, \Psi)$ and $(\mathbf{a}, \mathbf{e}, \psi) \in \mathrm{T}_{\mathbf{p}} \mathbf{P}, \mathrm{Eq}$. (1.12) implies that

$$
\left\langle\mathrm{dJ}_{\mathbf{p}}(\mathbf{a}, \mathbf{e}, \psi) \mid \xi\right\rangle=\int_{\mathbf{M}}\left\{-(\operatorname{div}(\mathbf{e})+[\mathbf{A}, \mathbf{e}]+[\mathrm{E}, \mathrm{a}]) \xi+\psi^{\dagger} \xi \Psi+\Psi^{\dagger} \xi \psi\right\} \mathrm{d}_{3} \mathrm{x} .
$$

Hence, $\mathrm{dJ}_{\mathbf{p}}=\dot{T}+S: \mathrm{T}_{\mathbf{p}} \mathbf{P} \rightarrow \mathrm{L}^{2}(\mathrm{M}, \mathfrak{g})$, where

$$
T(\mathbf{a}, \mathbf{e}, \psi)=-\operatorname{div}(\mathbf{e}) \quad \text { and } \quad S(\mathbf{a}, \mathbf{e}, \psi)=-[\mathbf{A}, \mathbf{e}]-[\mathrm{E}, \mathbf{a}]+\psi^{\dagger} \otimes \Psi+\Psi^{\dagger} \otimes \psi
$$

The Hodge decomposition, cf. [11], applied to square integrable zero forms on $M$, implies that $\mathrm{L}^{2}(\mathrm{M}, \mathfrak{g})=\mathcal{C} \oplus \mathcal{K}$, where $\mathcal{X}$ is the space of constant $\mathfrak{g}$-valued functions and $\mathcal{C}$ $=\left\{\operatorname{div}(\mathbf{v}) \mid \mathbf{v} \in \mathrm{H}^{1}(\mathbf{M}, \mathfrak{g}), \mathrm{nv}=0\right\}$. Both $\mathcal{C}$ and $\mathcal{X}$ are closed subspaces of $\mathrm{L}^{2}(\mathbf{M}, \mathfrak{g})$. Since range $T=\mathcal{C}$, it follows that the range of $T$ is closed. Moreover, cokernel $T=$ $\mathrm{L}^{2}(\mathrm{M}, \mathfrak{g}) /$ range $T \simeq \mathcal{X}$ has finite dimension, since $\operatorname{dim} \mathcal{X}=\operatorname{dim} \mathfrak{g}$. Hence, $T$ is semi-Fredholm.

Further, if $v_{n}=\left(a_{n}, e_{n}, \psi_{n}\right)$ is a bounded sequence in $T_{p} P$, then the sequence

$$
\left\{S \mathbf{v}_{n}\right\}=\left\{-\left[\mathrm{A}, \mathrm{e}_{\mathrm{n}}\right]-\left[\mathrm{E}, \mathrm{a}_{\mathrm{n}}\right]+\psi_{\mathrm{n}}^{\dagger} \otimes \Psi+\Psi^{\dagger} \otimes \psi_{\mathrm{n}}\right\}
$$

is bounded in $H^{1}(M, \mathfrak{g}), \subset L^{2}(M, \mathfrak{g})$. Since the embedding of $H^{1}(M, \mathfrak{g})$ into $L^{2}(M, \mathfrak{g})$ is compact, it follows that the sequence $\left\{S \mathbf{v}_{\mathrm{n}}\right\}$ has a convergent subsequence. That is, the operator $S$ is compact. This implies that $\mathrm{dJ}_{\mathrm{p}}=T+S$ is semi-Fredholm, that is it has closed range and finite codimension, c.f. [12].

Q.E.D. 
For each $\mathbf{p} \in \mathbf{P}$ we denote by $g s_{\mathbf{p}}$ the gauge symmetry (isotropy) algebra of $\mathbf{p}$, that is

$$
g s_{\mathrm{p}}=\left\{\xi \in g s(\mathrm{P}) \mid \xi_{\mathrm{P}}(\mathrm{p})=0\right\},
$$

and by $G S_{\mathrm{p}}$ gauge symmetry (isotropy) group of $\mathrm{p}$,

$$
G S_{\mathrm{p}}=\{\phi \in G S(\mathrm{P}) \mid \phi \mathrm{p}=\mathrm{p}\}
$$

By properness of the action of $G S(\mathbf{P})$ in $\mathbf{P}$, each sequence $\left\{\phi_{n}\right\}$ in $G S_{\mathbf{p}}$ has a convergent subsequence, which implies that $G S_{\mathrm{p}}$ is compact. Consequently, the Lie algebra $g s_{p}$ is finite dimensional. It is isomorphic to a subalgebra of the structure algebra $\mathfrak{g}$; a construction of such an ismomorphism is given in Section 5 .

The annihilator of the subalgebra $\mathfrak{h} \subseteq g s(\mathrm{P})$ is the subspace $\mathfrak{h}^{\mathfrak{a}} \subseteq g s(\mathrm{P})^{*}$ defined by

$$
\mathfrak{h}^{\mathrm{a}}=\left\{v \in g s(\mathrm{P})^{*} \mid\langle v \mid \xi\rangle=0 \forall \xi \in \mathfrak{h}\right\} \text {. }
$$

\section{PROPOSITION 2.4.}

The range of the map $\mathrm{dJ}_{\mathbf{p}}: \mathrm{T}_{\mathbf{p}} \mathbf{P} \rightarrow g s(\mathbf{P})$ is given by the annihilator of the symmetry algebra of $p$, that is

$$
\text { range } \mathrm{dJ}_{\mathrm{p}}=\left(g s_{\mathrm{p}}\right)^{\mathrm{a}} .
$$

Proof. By.(1.11), for each $\xi \in g s(\mathbf{P})$, and $\mathbf{p} \in \mathbf{P}$,

$$
\left\langle\mathrm{dJ}_{\mathbf{p}}(.) \mid \xi\right\rangle=\xi_{\mathbf{P}}(\mathrm{p}) \perp \omega .
$$

Since $\omega$ is non-degenerate, it follows from (2.9) that

$$
g s_{\mathbf{p}}=\left\{\xi \in g s(\mathbf{P}) \mid\left\langle\mathrm{dJ}_{\mathbf{p}}(\mathbf{v}) \mid \xi\right\rangle=0 \forall \mathbf{v} \in \mathrm{T}_{\mathbf{p}} \mathbf{P}\right\}=\left(\text { range } \mathrm{d} J_{\mathbf{p}}\right)^{\mathrm{a}} .
$$

Since range $\mathrm{dJ}_{\mathrm{p}}$ is closed, taking annihilators of both sides we obtain

$$
\left(g s_{\mathbf{p}}\right)^{\mathrm{a}}=\left(\text { range } \mathrm{dJ}_{\mathbf{p}}\right)^{\mathrm{aa}}=\text { range } \mathrm{dJ}_{\mathrm{p}},
$$

provided that (range $\mathrm{dJ}_{\mathbf{p}}$ ) ${ }^{\text {aa }}$ is the closure of range $\mathrm{dJ}_{\mathrm{p}}$.

In order to prove the last assertion, denote by $R_{p}$ the closure of range $\mathrm{dJ}_{\mathrm{p}}$ in the topological dual $g s(\mathbf{P})^{\prime}$ of $g s(\mathrm{P})$. The polar of $\mathrm{R}_{\mathrm{p}}$ is 
and the bi-polar

$$
\left(\mathrm{R}_{\mathbf{p}}\right)^{0}=\left\{\xi \in g s(\mathbf{P}) \mid\langle v \mid \xi\rangle=0 \forall v \in \mathbf{R}_{\mathbf{p}}\right\}
$$

$$
\left(\mathrm{R}_{\mathrm{p}}\right)^{00}=\left\{v \in g s(\mathrm{P})^{\prime} \mid\langle v \mid \xi\rangle=0 \forall \xi \in{ }^{\prime}\left(\mathrm{R}_{\mathrm{p}}\right)^{0}\right\}
$$

is the closure of $R_{p}$ in $g s(\mathbf{P})^{\prime}$, c.f. [13]. By definition $R_{p}^{\prime}$ is closed so that $R_{p}=\left(R_{p}\right)^{00}$. Since range $\mathrm{dJ}_{\mathrm{p}}$ is dense in $\mathrm{R}_{\mathrm{p}}$, it follows that

$$
\left(\mathrm{R}_{\mathrm{p}}\right)^{0}=\left(\text { range } \mathrm{dJ}_{\mathrm{p}}\right)^{\mathrm{a}}
$$

Hence,

$$
\left(\text { range } \mathrm{d}_{\mathrm{p}}\right)^{\mathrm{aa}}=\left(\mathrm{R}_{\mathrm{p}}\right)^{00} \cap g s(\mathrm{P})^{*}=\mathrm{R}_{\mathrm{p}} \mathrm{P} g s(\mathrm{P})^{*},
$$

which implies that (range $\left.\mathrm{dJ}_{/ \mathbf{p}}\right)^{\text {aa }}$ is the closure of range $\mathrm{dJ}_{\mathrm{p}}$ in $g s(\mathbf{P})^{*}$.

Q.E.D.

We conclude from Proposition 2.4 that $\mathrm{p}$ is a regular point of the momentum map $\mathrm{J}$ if and only if $\mathbf{p}$ has no infinitesimal symmetries, i.e. $g s_{\mathbf{p}}=\{0\}$. In this case $\mathrm{J}-1(\mathrm{~J}(\mathrm{p}))$ is a manifold in a neighbourhood of $p$ with the tangent space

$$
\mathrm{T}_{\mathrm{p}} \mathrm{J}-1(\mathrm{~J}(\mathrm{p}))=\text { ker } \mathrm{dJ}_{\mathrm{p}} \text {. }
$$

Singular points of the momentum map have non trivial algebras of infinitesimal . symmetries. Let $\mathfrak{h}$ be the Lie algebra of a connected compact subgroup $\mathrm{H}$ of $G S(\mathrm{P})$. We denote by $\mathbf{P}_{\mathfrak{h}}$ the set of points $\mathbf{p}$ in $\mathbf{P}$ such that $g s_{\mathbf{p}}=\mathfrak{h}$, that is

$$
\mathbf{P}_{\mathfrak{h}}=\left\{\mathbf{p} \in \mathbf{P} \mid g s_{\mathbf{p}}=\mathfrak{h}\right\} \text {. }
$$

It follows from Proposition 2.4 that $\mathbf{p} \in \mathbf{P}_{\mathfrak{h}}$ if and only if for all $\mathbf{v} \in T_{\mathbf{p}} \mathbf{P}$

$$
\left\langle\mathrm{dJ}_{\mathbf{p}}(\mathbf{v}) \mid \xi\right\rangle=0 \quad \forall \quad \xi \in \mathfrak{h} \quad \text { and }\left\langle\mathrm{dJ}_{\mathbf{p}}(\mathbf{v}) \mid \zeta\right\rangle \neq 0 ; \forall \quad \zeta \notin \mathfrak{h} .
$$

Let $\overline{\mathbf{P}}_{\mathfrak{h}}$ be the set of points in $\mathbf{P}$ such that their symmetry algebra $g s_{\mathbf{p}}$ contains $\mathfrak{h}$, i.e.

$$
\overline{\mathbf{P}}_{\mathfrak{h}}=\left\{\mathbf{p} \in \mathbf{P} \mid \mathfrak{h} \subseteq g s_{\mathbf{p}}\right\}
$$

It is clear that $\overline{\mathbf{P}}_{\mathfrak{h}}=\underset{\mathfrak{k} \supseteq \mathfrak{h}}{\cup} \mathbf{P}_{\mathfrak{k}}$. 


\section{Proposition 2.5.}

(1) $\overline{\mathbf{P}}_{\mathfrak{h}}$ is a closed affine subspace of $\mathbf{P}$.

(2) $P_{\mathfrak{h}}$ is a submanifold of $\mathbf{P}$ with the tangent space

$$
\mathrm{T}_{\mathbf{p}} \mathbf{P}_{\mathfrak{h}}=\left\{\mathbf{v} \in \mathrm{T}_{\mathbf{p}} \mathbf{P} \mid\left\langle\mathrm{D}^{2} \mathrm{~J}_{\mathbf{p}}(\mathbf{u}, \mathbf{v}) \mid \xi\right\rangle=0, \forall \quad \mathbf{u} \in \mathrm{T}_{\mathbf{p}} \mathbf{P}_{\mathfrak{h}}, \boldsymbol{\xi} \in \mathfrak{h}\right\}:
$$

(3) The restrictions of the symplectic form $\omega$ to $\overline{\mathbf{P}}_{\mathfrak{h}}$ and $\mathbf{P}_{\mathfrak{h}}$ are symplectic.

\section{PROOF.}

(1) It follows from the fact that the action of $G S(\mathbf{P})$ in $\mathbf{P}$ is continuous and affine.

(2) Let $\mathbf{p}$ be a point in $\mathbf{P}_{\mathfrak{h}}$. Then, for every $\zeta \notin \mathfrak{h}$, the linear map $\left\langle\mathrm{dJ}_{\mathbf{p}}() \mid. \zeta\right\rangle$ : $\mathrm{T}_{\mathbf{p}} \mathbf{P} \rightarrow \mathbb{R}$ does not vanish identically. Since $\mathrm{dJ}$ is continuous, there exists a neighbourhood $\mathbf{U}$ of $\mathbf{p}$ in $\mathbf{P}$ such that, for all $\mathbf{q} \in \mathrm{U},\left\langle\mathrm{dJ}_{\mathbf{q}}() \mid. \zeta\right\rangle \neq 0$ for every $\zeta$ not in $\mathfrak{h}$. This implies that $\mathbf{U} \cap \overline{\mathbf{P}}_{\mathfrak{h}} \subseteq \mathbf{P}_{\mathfrak{h}}$. Hence, $\mathbf{P}_{\mathfrak{h}}$ is an open submanifold of $\overline{\mathbf{P}}_{\mathfrak{h}}$ and a submanifold of $\mathbf{P}$. The expression (2.18) for the tangent space of $\mathbf{P}_{\mathfrak{h}}$ is obtained by differentiating $\left\langle\mathrm{dJ}_{\mathbf{p}}() \mid. \xi\right\rangle=0$ for all $\xi \in \mathfrak{h}$ and all $\mathbf{p} \in \mathbf{P}_{\mathfrak{h}}$.

(3) Here we have to use the almost complex structure described in Appendix A. Let $\tilde{\mathbf{P}}$ be the $\mathrm{L}^{2}$-closure of $\mathbf{P}$. Then $\tilde{\mathbf{P}}_{\mathfrak{h}}=\left\{\mathbf{p} \in \tilde{\mathbf{P}} \mid \mathfrak{h} \subseteq g s_{\mathbf{p}}\right\}$ is the L2-closure of $\overline{\mathbf{P}}_{\mathfrak{h}}$. Since $\mathcal{J}$ is $G S(\mathbf{P})$ invariant, it follows that $\tilde{\mathfrak{P}}_{\mathfrak{h}}=\tilde{\mathbf{P}}_{\mathfrak{h}}$. Suppose that $\mathbf{u} \in \mathbf{T} \overline{\mathbf{P}}_{\mathfrak{h}}$ is such that $\mathbf{u}$ is in the kernel of $\omega \mid \overline{\mathbf{P}}_{\mathfrak{h}}$. Since $T \overrightarrow{\mathbf{P}}_{\mathfrak{h}} \subset T \tilde{\mathbf{P}}_{\mathfrak{h}}$,

$$
\langle\mathcal{J u} \mid \mathbf{v}\rangle_{\mathrm{L}^{2}}=\tilde{\omega}(\mathbf{u}, \mathbf{v})=\omega(\mathbf{u}, \mathbf{v})=0
$$

for every $\mathbf{v} \in T \overline{\mathbf{P}}_{\mathfrak{h}}$. By construction $T \overline{\mathbf{P}}_{\mathfrak{h}}$ is dense in $T \tilde{\mathbf{P}}_{\mathfrak{h}}$, hence $\mathcal{J} \mathbf{u}=0$, and $\mathbf{u}=0$. Therefore, $\overline{\mathbf{P}}_{\mathfrak{h}}$ is symplectic. Since $\mathbf{P}_{\mathfrak{h}}$ is an open submanifold of $\overline{\mathbf{P}}_{\mathfrak{h}}$ it is also symplectic.

Q.E.D.

The last essential property of the action of $G S(P)$ in $P$ needed here is the existence of slices. A slice through a point $p \in \mathbf{P}$ for the action of $G S(\mathrm{P})$ is a submanifold $S_{p}$ of $\mathbf{P}$ containing $\mathbf{p}$, and such that 
(1) $S_{p}$ is transverse and complementary to the orbit $O_{\mathbf{p}}$ at $\mathrm{p}$, that is

$$
\mathrm{T}_{\mathbf{p}} \mathbf{S}_{\mathrm{p}} \oplus \mathrm{T}_{\mathbf{p}} O_{\mathrm{p}}=\mathrm{T}_{\mathbf{p}} \mathbf{P}
$$

(2) $\mathrm{S}_{\mathrm{p}}$ is transverse to all $G S(\mathrm{P})$ orbits, that is, for each $\mathrm{q} \in \mathrm{S}_{\mathrm{p}}$,

$$
\mathrm{T}_{\mathrm{q}} \mathrm{S}_{\mathrm{p}}+\mathrm{T}_{\mathrm{q}} \mathrm{O}_{\mathrm{q}}=\mathrm{T}_{\mathrm{q}} \mathrm{P}
$$

(3) $\mathrm{S}_{\mathrm{p}}$ is invariant under the action of the gauge symmetry group $G S_{\mathrm{p}}$ of $\mathbf{p}$.

(4) For $\mathbf{q} \in \mathbf{S}_{\mathbf{p}}$ and $\phi \in G S(\mathbf{P})$, if $\phi \mathbf{q} \in \mathrm{S}_{\mathbf{p}}$ then $\phi \in G S_{\mathbf{p}}$.

THEOREM 2 (Slice Theorem).

For each $\mathbf{p} \in \mathbf{P}$ there exists a slice $S_{\mathbf{p}}$ through $\mathbf{p}$ for the action of $G S(\mathrm{P})$ such that $\mathrm{T}_{\mathbf{p}} \mathbf{S}_{\mathbf{p}}$ is $\mathrm{L}^{2}$ orthogonal to $\mathrm{T}_{\mathbf{p}} O_{\mathrm{p}}$.

ProoF is given in Appendix $C$.

3. Structure of the constraint set.

The constraint set is the zero level of the momentum map J. It follows from Proposition 2.4 that $\mathrm{J}^{-1}(0)$ need not be a manifold in neighbourhoods of points admitting infinitesimal symmetries. We shall show that it is foliated by submanifolds labelled by the Lie algebras $\mathfrak{h}$ of compact subgroups of $G S(\mathbf{P})$. Let $\mathbf{M}_{\mathfrak{h}}$ be the intersection of $\mathrm{J}^{-1}(0)$ with the submanifold $\mathbf{P}_{\mathfrak{h}}$,

$$
\mathbf{M}_{\mathfrak{h}}=\mathrm{J}^{-1}(0) \cap \mathbf{P}_{\mathfrak{h}}
$$

We denote by $\mu_{\mathfrak{h}}$ the pull-back of $\omega$ by the inclusion map $j_{\mathfrak{h}}: \mathbf{M}_{\mathfrak{h}} \rightarrow \mathbf{P}$,

$$
\mu_{\mathfrak{h}}=\mathrm{j}_{\mathfrak{h}}^{*} \omega
$$

By $\mathfrak{n}(\mathfrak{h})$ we denote the normaliser of $\mathfrak{h}$ in $g s(\mathrm{P})$,

$$
\mathfrak{n}(\mathfrak{h})=\{\xi \in g s(\mathbf{P}) \mid[\xi, \zeta] \in \mathfrak{h} \forall \zeta \in \mathfrak{h}\} .
$$




\section{THEOREM 3.}

For every compact connected subgroup $\mathrm{H}$ of $G S(\mathbf{P})$, with Lie algebra $\mathfrak{h},\left(\mathbf{M}_{\mathfrak{h}}, \mu_{\mathfrak{h}}\right)$ is a co-isotropic submanifold of $(\mathbf{P}, \omega)$. The null distribution of $\mu_{\mathfrak{h}}$ is spanned by the vector fields $\xi_{\mathrm{P}}$, for $\xi \in \mathfrak{n}(\mathfrak{h})$.

The proof of this theorem will be given in a series of propositions. First, we observe that the annihilator $\mathfrak{h}^{\mathrm{a}}$ is closed, and hence

$$
g s(\mathbf{P})^{*}=\mathfrak{h}^{\mathrm{a}} \oplus\left(\mathfrak{h}^{\mathrm{a}}\right)^{\perp} .
$$

We denote by $\pi_{\mathfrak{h}}: g s(\mathrm{P})^{*} \rightarrow \mathfrak{h}^{\text {a }}$ the projections on the first component, and by $\mathrm{K}$ the composition of $\mathrm{J}$ with $\pi_{\mathfrak{h}}$,

$$
\mathrm{K}=\pi_{\mathfrak{h}} \circ \mathrm{J}: \mathbf{P} \rightarrow \mathfrak{h}^{\mathrm{a}} .
$$

It should be noted that the map $\mathrm{K}$ depends on the choice of the infinitesimal symmetry algebra $\mathfrak{h}$, but we shall not label it by the subscript $\mathfrak{h}$ in order to simplify the notation. Moreover, $\mathrm{J}^{-1}(0) \subseteq \mathrm{K}^{-1}(0)$ for every $\mathfrak{h}$, so that

$$
\mathbf{M}_{\mathfrak{h}}=J^{-1}(0) \cap \mathbf{P}_{\mathfrak{h}} \subseteq \mathrm{K}^{-1}(0) \cap \mathbf{P}_{\mathfrak{h}} .
$$

\section{Proposition 3.1.}

For every $\mathrm{p} \in \mathrm{K}^{-1}(0) \cap \mathbf{P}_{\mathfrak{h}}, \mathrm{K}^{-1}(0)$ is a submanifold of $\mathbf{P}$ in a neighbourhood of $\mathbf{p}$ and

$$
\mathrm{T}_{\mathbf{p}} \mathrm{K}^{-1}(0)=k e r \mathrm{dJ}_{\mathbf{p}}
$$

Proof. Since $\mathrm{p} \in \mathrm{P}_{\mathfrak{h}} \cap \mathrm{K}^{-1}(0)$, it follows from Prop. 2.4 that

$$
\text { range } \left.\mathrm{dK}_{\mathrm{p}}=\pi_{\mathfrak{h}} \text { (range } \mathrm{dJ}_{\mathbf{p}}\right)=\pi_{\mathfrak{h}}\left(\mathfrak{h}^{\mathrm{a}}\right)=\mathfrak{h}^{\mathrm{a}} \text {, }
$$

which implies that $K$ is a submersion. Hence, $K^{-1}(0)$ is locally a submanifold of $P$. Moreover, $\mathrm{T}_{\mathbf{p}} \mathrm{K}^{-1}(0)=\operatorname{ker} \mathrm{dK}_{\mathrm{p}}=\operatorname{ker} \mathrm{d}\left(\pi_{\mathfrak{h}} \circ{ }^{\circ J}\right)=k e r \mathrm{dJ}_{\mathrm{p}}$. 


\section{Proposition 3.2.}

$$
\begin{gathered}
\mathrm{K}^{-1}(0) \cap \mathbf{P}_{\mathfrak{h}} \text { is a submanifold of } \mathbf{P}_{\mathfrak{h}} . \text { For every } \mathbf{p} \in \mathrm{K}^{-1}(0) \cap \mathbf{P}_{\mathfrak{h}}, \\
\mathrm{T}_{\mathbf{p}}\left(\mathrm{K}^{-1}(0) \cap \mathbf{P}_{\mathfrak{h}}\right)=\left\{\mathbf{v} \in \operatorname{ker} \mathrm{dJ}_{\mathbf{p}} \mid\left\langle\mathrm{D}^{2} \mathrm{~J}_{\mathbf{p}}(\mathbf{v}, \mathbf{w}) \mid \xi\right\rangle=0 \forall \xi \in \mathfrak{h}, \mathbf{w} \in \mathrm{T}_{\mathbf{p}} \mathbf{P}\right\}
\end{gathered}
$$

Proof. For every $\mathbf{p} \in \mathrm{K}^{-1}(0) \cap \mathbf{P}_{\mathfrak{h}}, \mathbf{K}$ is a submersion in a neighbourhood of $\mathbf{p}$. Hence, for every $v \in h^{a}$, there exists a vector field $X_{v}$ in $P$ such that $v=d K\left(X_{v}\right)=$ $\pi_{\mathfrak{h}}\left(\mathrm{dJ}\left(\mathrm{X}_{v}\right)\right)$ in a neighbourhood of $\mathrm{p}$. Also, $v \in \mathfrak{h}^{\mathrm{a}}$ implies that $\left\langle\mathrm{dJ}\left(\mathrm{X}_{v}\right) \mid \xi\right\rangle=0$ for. all $\xi \in \mathfrak{h}$. Differentiating this equation in direction of $w \in T_{\mathbf{p}} \mathbf{P}$ we obtain

$$
\left\langle\mathrm{D}^{2} \mathrm{~J}_{\mathrm{p}}\left(\mathrm{w}, \mathrm{X}_{v}(\mathrm{p})\right) \mid \xi\right\rangle+\left\langle\mathrm{dJ}_{\mathbf{p}}\left(\mathrm{DX}{ }_{v}(\mathbf{w})\right) \mid \xi\right\rangle=0 .
$$

However, $\left\langle\mathrm{dJ}_{\mathbf{p}}\left(\mathrm{DX} \mathrm{v}_{v}(\mathbf{w})\right) \mid \xi\right\rangle=0$ since $\left\langle\mathrm{dJ}_{\mathbf{p}}() \mid. \xi\right\rangle=0$ for all $\xi \in \mathfrak{h}$. Hence, $\left\langle\mathrm{D}^{2 \mathrm{~J}} \mathrm{p}_{\mathbf{p}}\left(\mathbf{w}, \mathrm{X}_{v}(\mathbf{p})\right) \mid \xi\right\rangle=0$ for all $\xi \in \mathfrak{h}$, which implies by Eq. (2.18) that $\mathrm{X}_{v}(\mathrm{p}) \in \mathrm{T}_{\mathbf{p}} \mathbf{P}_{\mathfrak{h}}$. Therefore, the restriction of $K$ to $P_{\mathfrak{h}}$ is a submersion at $\mathbf{p}$, and $K^{-1}(0) \cap \mathbf{P}_{\mathfrak{h}}=$ $\left(K \mid P_{\mathfrak{h}}\right)^{-1}(0)$ is a submanifold of $\mathbf{P}_{\mathfrak{h}}$. Eq. (3.8) follows from (3.7).

Q.E.D.

Most of the following analysis is local. Therefore, we fix a point $\mathrm{p}_{0}$ in $J^{-1}(0) \cap . P_{\mathfrak{h}}$ and discuss the structure of $\mathbf{M}_{\mathfrak{h}}$ in its neighbourhood.

Let $\mathrm{S}_{\mathrm{p}_{0}}$ be a slice for the $G S(\mathrm{P})$ action such that ${ }_{\mathrm{p}_{0}}^{\mathrm{T}} \mathrm{S}_{\mathrm{p}_{0}}$ is the $\mathrm{L}^{2}$ orthogonal complement of $\mathrm{T}_{\mathrm{p}_{0}} \mathrm{O}_{\mathrm{p}_{0}}$,

$$
\mathrm{T}_{\mathrm{p}_{0}} \mathrm{~S}_{\mathrm{p}_{0}}=\left(\mathrm{T}_{\mathrm{p}_{0}} O_{\mathrm{p}_{0}}\right)^{\perp}
$$

\section{PropositTon 3.3.}

$$
\begin{gathered}
\mathrm{P}_{\mathfrak{h}} \cap \mathrm{S}_{\mathrm{p}_{0}} \text { is a manifold in a neighbourhood of } \mathrm{p}_{0} \text { with tangent space } \\
\mathrm{T}_{\mathrm{p}_{0}}\left(\mathrm{P}_{\mathfrak{h}} \cap S_{\mathrm{p}_{0}}\right)=\left\{\mathrm{v} \in\left(\mathrm{T}_{\mathrm{p}_{0}} O \mathrm{p}_{0}\right)^{\perp} \mid\left\langle\mathrm{D}^{2} \mathrm{~J}(\mathbf{v}, \mathbf{w}) \mid \xi\right\rangle=0 \forall \xi \in \mathfrak{h} ; \mathbf{w} \in \mathrm{T}_{\mathrm{p}_{0}} \mathrm{P}\right\}
\end{gathered}
$$

Proof. Let $\mathbf{P}_{(\mathfrak{h})}$ the set of points on the $G S(\mathbf{P})$ orbits through $\mathbf{P}_{\mathfrak{h}}$, that is

$$
\mathbf{P}_{(\mathfrak{h})}=\left\{\phi \mathbf{q} \mid \phi \in G S(\mathbf{P}), \mathbf{q} \in \mathbf{P}_{\mathfrak{h}}\right\}
$$


If $\psi \mathbf{q}=\mathbf{p}$, and $\mathbf{q} \in \mathbf{P}_{\mathfrak{h}}$, then $\psi \phi \psi^{-1} \mathbf{p}^{\prime}=\mathbf{p} \quad \forall \phi \in \mathrm{H}$. Thus, for every $\mathbf{q} \in \mathbf{P}_{(\mathfrak{h})}$, the symmetry group $G S_{\mathbf{q}}$ is conjugate to $\mathrm{H}$. Moreover, property $(4)$ of a slice yields

$$
\begin{gathered}
\mathbf{P}_{(\mathfrak{h})} \cap S_{\mathrm{p}}=\left\{\phi \mathbf{q} \in S_{\mathbf{p}} \mid \phi \in G S(\mathbf{P}), \mathbf{q} \in \mathbf{P}_{\mathfrak{h}}\right\}= \\
=\left\{\phi \mathbf{q} \in S_{\mathbf{p}} \mid \phi \in \mathrm{H}, \mathbf{q} \in \mathbf{P}_{\mathfrak{h}}\right\}=\mathbf{P}_{\mathfrak{h}} \cap S_{\mathbf{p}} .
\end{gathered}
$$

By definition of $\mathbf{P}_{(\mathfrak{h})}$ and property $2 /$ of the slice, $\mathbf{P}_{(\mathfrak{h})}$ and $S_{\mathbf{p}_{0}}$ are transverse at $\mathbf{p}_{0}$. It follows that $\mathbf{P}_{\mathfrak{h}} \cap S_{\mathbf{p}_{0}}$ is a submanifold of $S_{p_{0}}$ in a neighbourhood of $\mathbf{p}_{0}$. Further, Eq. (2.16), implies that

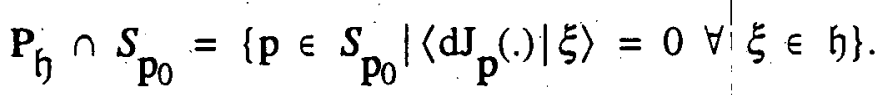

Differentiating this condition at $\mathrm{p}_{0}$ and taking into account Eq. (3.9) we obtain (3.10).

Q.E.D.

PROPOSITION 3.4.

$\mathbf{M}_{\mathfrak{h}}$ is an open subset of $\mathbf{P}_{\mathfrak{h}} \cap \mathrm{K}^{-1}(0)$, and

$$
\mathbf{M}_{\mathfrak{h}} \cap S_{\mathrm{p}_{0}}=\mathrm{P}_{\mathfrak{h}} \cap S_{\mathrm{p}_{0}} \cap \mathrm{K}^{-1}(0)
$$

Proof. By Eq. (3.12), $\left\langle\mathrm{dJ}_{\mathbf{p}^{(.)}} \mid \xi\right\rangle=0$ for all $\mathrm{p} \in \mathrm{P}_{\mathfrak{h}} \cap S_{\mathrm{p}_{0}} \cap \mathrm{K}^{-1}(0)$ and all $\xi \in \mathfrak{h}$. Therefore, the function $\langle\mathrm{J} \mid \xi\rangle$ on $\mathrm{P}$ is constant on connected components of $\mathrm{P}_{\mathfrak{G}} \cap S_{\mathrm{p}_{0}} \cap \mathrm{K}^{-1}(0)$. By hypothesis $\mathrm{J}\left(\mathrm{p}_{0}\right)=0$. Therefore $\langle\mathrm{J}(\mathrm{p}) \mid \xi\rangle=0$ for all $\mathrm{p}$ in the connected component of $\mathbf{P}_{\mathfrak{h}} \cap S_{\mathbf{p}_{0}} \cap \mathrm{K}^{-1}(0)$ containing $\mathbf{p}_{0}$. Hence, $\mathrm{J} \mid \mathbf{P}_{\mathfrak{h}} \cap S_{\mathbf{p}_{0}} \cap \mathrm{K}^{-1}(0)$ has range in $\mathfrak{h}$. By. definition $\mathrm{K}=\pi_{\mathfrak{h}} \circ \mathrm{J}: \mathrm{P} \rightarrow \mathfrak{h}^{\mathrm{a}}$, so that $\mathrm{J}\left(\mathrm{P}_{\mathfrak{h}} \cap S_{\mathrm{p}_{0}} \cap \mathrm{K}^{-1}(0)\right)=$ $\mathrm{K}\left(\mathrm{P}_{\mathfrak{h}} \cap S_{\mathrm{P}_{0}} \cap \mathrm{K}^{-1}(0)\right)=0$. Since $\mathrm{M}_{\mathfrak{h}}=\mathrm{P}_{\mathfrak{h}} \cap \mathrm{J}^{-1}(0)$ and $\mathrm{J}-1(0) \subseteq \mathrm{K}^{-1}(0)$, it follows that

$$
\dot{\mathbf{M}}_{\mathfrak{h}} \cap S_{\mathrm{p}_{0}}=\mathbf{P}_{\mathfrak{h}} \cap S_{\mathrm{p}_{0}} \cap \mathrm{K}^{-1}(0) \text {. }
$$

The preceding local analysis is valid for every $p_{0} \varepsilon \in P_{\mathfrak{h}} \cap J^{-1}(0)$. In order to show that $\mathbf{M}_{\mathfrak{h}}$ is open in $\mathbf{P}_{\mathfrak{h}} \cap \mathrm{K}^{-1}(0)$, for each $\mathbf{p}_{0} \in \mathbf{P}_{\mathfrak{h}} \cap \mathrm{J}^{-1}(0)$, we choose a slice $S_{\mathrm{p}_{0}}$ through $\mathrm{p}_{0}$ satisfying (3.9), and a neighbourhood $\mathrm{V}_{\mathrm{p}_{0}}$ of $\mathrm{p}_{0}$ in $O_{\mathrm{p}_{0}}$ such that 
$S_{\mathrm{p}_{0}} \times \mathrm{V}_{\mathrm{p}_{0}}$ is an open neighbourhood of $\mathrm{p}_{0}$ in $\mathrm{P}$. The collection

$$
\left\{\left(S_{\mathbf{p}_{0}} \times \mathrm{V}_{\mathrm{p}_{0}}\right) \cap \mathbf{P}_{\mathfrak{h}} \cap \mathrm{K}^{-1}(0) \mid \mathbf{p}_{0} \in \mathrm{M}_{\mathfrak{h}}\right\}
$$

is an open covering of $\mathbf{M}_{\mathfrak{h}}$ in $\mathbf{P}_{\mathfrak{h}} \cap \mathrm{K}^{-1}(0)$. If $\mathbf{p} \in \mathrm{P}_{\mathfrak{h}} \cap \mathrm{K}^{-1}(0)$ is contained in $\left(S_{\mathbf{p}_{0}} \times \mathrm{V}_{\mathbf{p}_{0}}\right)$, for some $\mathrm{p}_{0} \in \mathbf{M}_{\mathfrak{h}}$, then there exists $\phi \in G S(\mathbf{P})$ such that $\mathbf{p}$ is contained in the slice $S_{\phi \mathrm{p}_{0}}^{\prime}=\phi\left(S_{\mathrm{p}_{0}}\right)$ through $\phi \mathrm{p}_{0}$, satisfying (3.9), which need not to belong to the collection of slices chosen in (3.14). Hence,

$$
\mathbf{p} \in \mathrm{P}_{\mathfrak{h}} \cap S_{\phi \mathrm{p}_{0}}^{\prime} \cap \mathrm{K}^{-1}(0)=\mathbf{P}_{\mathfrak{h}} \cap S_{\phi \mathrm{p}_{0}}^{\prime} \cap \mathrm{J}^{-1}(0) \subseteq \mathbf{P}_{\mathfrak{h}} \cap \mathrm{J}^{-1}(0)=\mathbf{M}_{\mathfrak{h}} .
$$

This implies that the union of sets in (3.14) is contained in $\mathbf{M}_{\mathfrak{h}}$. Hence, $\mathbf{M}_{\mathfrak{h}}$ is open in $\mathbf{P}_{\mathfrak{h}} \cap \mathrm{K}^{-1}(0)$.

Q.E.D.

\section{PROPOSTTION 3.5}

$\mathbf{M}_{\mathfrak{h}} \cap S_{\mathbf{p}_{0}}$ is a symplectic manifold in a neighbourhood of $\mathrm{p}_{0}$ with tangent space

$$
\begin{gathered}
\mathrm{T}_{\mathbf{p}_{0}}\left(\mathbf{M}_{\mathfrak{h}} \cap \mathrm{S}_{\mathbf{p}_{0}}\right)= \\
=\left\{\mathbf{v} \in\left(\mathrm{T}_{\mathbf{p}_{0}} O_{\mathbf{p}_{0}}\right)^{\perp} \cap k e r d \mathrm{dJ}_{\mathrm{p}_{0}} \mid\left\langle\mathrm{D}^{2} \mathrm{~J}(\mathbf{v}, \mathbf{w}) \mid \xi\right\rangle=0 \forall \xi \in \mathfrak{h}, \dot{w} \in \mathrm{T}_{\mathbf{p}_{0}} \mathbf{P}\right\} .
\end{gathered}
$$

Proof. Since $\mathbf{M}_{\mathfrak{h}} \cap \mathrm{S}_{\mathbf{p}_{0}}=\mathbf{P}_{\mathfrak{h}} \cap \mathrm{S}_{\mathbf{p}_{0}} \cap \mathrm{K}^{-1}(0)$, we prove the statement for $\mathbf{P}_{\mathfrak{h}} \cap \mathrm{S}_{\mathbf{p}_{0}} \cap$ $\mathrm{K}^{-1}(0)$. Consider the restriction $K \mid \mathbf{P}_{\mathfrak{h}} \cap \mathrm{S}_{\mathbf{p}_{0}}$. For each $\mathbf{v} \in \mathrm{T}_{\mathrm{p}_{0}} \mathbf{P}$, $\mathrm{dK}_{\mathrm{p}_{0}}(\mathrm{v})=\pi_{\mathfrak{h}}\left(\mathrm{dJ}_{\mathbf{p}_{0}}(\mathbf{v})\right)$. Hence, using Eq. (3.10),

$$
\begin{gathered}
\text { range } \mathrm{d}\left(\mathrm{K} \mid\left(\mathbf{P}_{\mathfrak{h}} \cap \mathrm{S}_{\mathbf{p}_{0}}\right)\right) \mathrm{p}_{0}= \\
=\left\{\pi_{\mathfrak{h}}\left(\mathrm{dJ}_{\mathrm{p}_{0}}(\mathrm{v})\right) \mid \mathrm{v} \in\left(\mathrm{T}_{\mathrm{p}_{0}} O_{\mathrm{p}_{0}}\right)^{\perp} \text { and }\left\langle\mathrm{D}^{2} \mathrm{~J}(\mathbf{v}, \mathbf{v}) \mid \xi\right\rangle=0 \mid \forall, \xi \in \mathfrak{h}, \mathbf{w} \in \mathrm{T}_{\mathrm{p}_{0}} \mathrm{P}\right\} .
\end{gathered}
$$

Since $\mathrm{T}_{\mathrm{p}_{0}} O_{\mathrm{p}_{0}} \subseteq$ ker $\mathrm{dK}_{\mathrm{p}_{0}}$, the condition $\mathbf{v} \in\left(\mathrm{T}_{\mathrm{p}_{0}} O_{\mathrm{p}_{0}}\right)^{\perp}$ can be omitted and, using Proposition 2.5, we obtain

$$
\begin{gathered}
\text { range } \mathrm{d}\left(\mathrm{K} \mid\left(\mathbf{P}_{\mathfrak{h}} \cap S_{\mathbf{p}_{0}}\right){ }_{\mathrm{p}_{0}}=\right. \\
\left\{\pi_{\mathfrak{h}}\left(\mathrm{dJ}{ }_{\mathbf{p}_{0}}(\mathbf{v})\right) \mid \mathbf{v} \in \mathrm{T}_{\mathbf{p}_{0}} \mathbf{P}_{\mathfrak{h}} \text { and }\left\langle\mathrm{D}^{2} J(\mathbf{v}, \mathbf{w}) \mid \xi\right\rangle=0 \forall \xi \in \mathfrak{h} ; \mathbf{w} \in \mathrm{T}_{\mathbf{p}_{0}} \mathbf{P}\right\}= \\
=\left\{\pi_{\mathfrak{h}}\left(\mathrm{dJ}_{\mathbf{p}_{0}} \mathbf{v}\right) \mid \mathbf{v} \in \mathrm{T}_{\mathbf{p}_{0}} \mathrm{P}_{\mathfrak{h}}\right\}=\text { range } \mathrm{d}\left(\mathrm{K}^{\prime} \mid \mathbf{P}_{\mathfrak{h}}\right) \mathbf{p}_{0} .
\end{gathered}
$$

In the proof of Prop. 3.2 we have shown that $K \mid \mathbf{P}_{\mathfrak{h}}$ is a submersion at $\mathbf{p}_{0}$. Hence, 
$\mathbf{K} \mid\left(\mathbf{P}_{\mathfrak{h}} \cap S_{\mathbf{p}_{0}}\right)$ is a submersion at $\mathbf{P}_{0}$, which implies that $\mathbf{P}_{\mathfrak{h}} \cap S_{\mathbf{p}_{0}} \cap \mathbf{K}^{-1}(0)=\left(K \mid\left(\mathbf{P}_{\mathfrak{h}} \cap\right.\right.$ $\left.S_{\mathrm{p}_{0}}\right)^{-1(0)}$ is locally a submanifold of $\mathbf{P}_{\mathfrak{h}} \cap S_{\mathbf{p}_{0}}$. The expression (3.15) for the tangent space follows from (3.7) and (3.10).

Let $\mathbf{v} \in \mathrm{T}_{\mathrm{p}_{0}}\left(\mathrm{P}_{\mathfrak{h}} \cap S_{\mathrm{p}_{0}} \cap \mathrm{J}^{-1}(0)\right)$. Since $\mathbf{v} \in \mathrm{T}_{\mathrm{p}_{0}}\left(\mathrm{P}_{\mathfrak{h}} \cap S_{\mathrm{p}_{0}}\right)$, Eqs. (3.10), (2.7) and (A.7), imply that $-\mathcal{J} \mathbf{v} \in J\left(\left(\mathrm{~T}_{\mathrm{p}_{0}} O_{\mathrm{p}_{0}}\right)^{\perp}\right)=k e r \mathrm{dJ}_{\mathrm{p}_{0}}$, where $\mathcal{J}$ is the almost complex structure discussed in Appendix A. Also, $\mathbf{v} \in$ ker $\mathrm{dJ}_{\mathrm{p}_{0}}$, so that $\mathcal{J} \mathbf{v} \in \mathcal{J}\left(k e r \mathrm{dJ}_{\mathrm{p}_{0}}\right)=$ $\left(\mathrm{T}_{\mathrm{p}_{0}} O_{\mathrm{p}_{0}}\right)^{\perp}$. Moreover, Lemma A.1 yields $\left\langle\mathrm{D}^{2} \mathrm{~J}_{\mathrm{p}_{0}}(\mathcal{J} \mathrm{v}, \mathcal{J} \mathbf{w}) \mid \xi\right\rangle=\left\langle\mathrm{D}^{2 \mathrm{~J}} \mathrm{p}_{0}(\mathrm{v}, \dot{w}) \mid \xi\right\rangle=0$ for all $\xi \in \mathfrak{h}, \mathbf{w} \in \mathrm{T}_{\mathbf{p}_{0}} \mathrm{P}$. Hence, $J_{\mathbf{v}} \in \mathrm{T}_{\mathrm{p}_{0}}\left(\mathrm{P}_{\mathfrak{h}} \cap \boldsymbol{S}_{\mathrm{p}_{0}} \cap \mathrm{K}^{-1}(0)\right)$, cf. Eq. (3.15).

Let $\mathbf{v} \in \mathrm{T}_{\mathrm{p}_{0}}\left(\mathbf{P}_{\mathfrak{h}} \cap S_{\mathrm{p}_{0}} \cap \mathrm{K}^{-1}(0)\right)$ be such that $\omega(\mathbf{v}, \mathbf{w})=0$ for all $\mathbf{w} \cdot \epsilon$ $\mathrm{T}_{\mathrm{p}_{0}}\left(\mathrm{P}_{\mathfrak{h}} \cap S_{\mathrm{p}_{0}} \cap \mathrm{K}^{-1}(0)\right)$. Then $\tilde{\omega}(\mathbf{v}, \tilde{\mathbf{w}})=0$ for all $\tilde{\mathrm{w}}$ in the $\mathrm{L}^{2}$ closure of $\mathrm{T}_{\mathrm{p}_{0}}\left(\mathbf{P}_{\mathfrak{h}} \cap S_{\mathrm{p}_{0}} \cap \mathrm{K}^{-1}(0)\right)$. Taking, $\tilde{\mathbf{w}}=\mathcal{J} \mathbf{v}$ we obtain $\|\mathbf{v}\|_{\mathrm{L}^{2}}^{2}=\langle\mathbf{v} \mid \dot{\mathbf{v}}\rangle_{\mathrm{L}^{2}}=\tilde{\omega}(\mathcal{J}, \mathbf{v})=0$. Hence, $\mathbf{v}=0$, which implies that $\mathrm{T}_{\mathrm{p}_{0}}\left(\mathrm{P}_{\mathfrak{h}} \cap S_{\mathrm{p}_{0}} \cap \mathrm{K}^{-1}(0)\right)$ is symplectic. Hence, $\mathbf{P}_{\mathfrak{h}} \cap S_{\mathrm{p}_{0}} \cap \mathrm{K}^{-1}(0)$ is symplectic in a neighbourhood of $\mathrm{p}_{0}$.

Q.E.D.

We have shown in Proposition 3.4 that, $\mathbf{M}_{\mathfrak{h}}$ is an open submanifold of $\mathbf{P}_{\mathfrak{h}} \cap \mathrm{K}^{-1}(0)$, the manifold structure of which has been established in Proposition 3.2. Moreover, $\mathbf{M}_{\mathfrak{h}} \cap S_{\mathbf{p}_{0}}$ is symplectic by Proposition 3.6. It remains to show that the pull-back $\mu_{\mathfrak{h}}$ of $\omega$ to $\mathbf{M}_{\mathfrak{h}}$ is co-isotropic with the null spanned by the vector fields $\xi_{\mathrm{P}}$, for $\xi$ in the normaliser $\mathfrak{n}(\mathfrak{h})$ of $\mathfrak{h}$, given by (3.3).

Let $\mathrm{N}(\mathrm{H})$ denote the normaliser of $\mathrm{H}$ in $G S(\mathrm{P})$; that is

$$
\mathrm{N}(\mathrm{H})=\left\{\phi \in G S(\mathrm{P}) \mid \phi^{-1} \chi \phi \in \mathrm{H} \forall \dot{\chi} \in \mathrm{H}\right\},
$$

and $\mathrm{N}_{0}(\mathrm{H})$ be the connected component of the identity in $\mathrm{N}(\mathrm{H})$. It is a subgroup of $G S(P)$ with the Lie algebra $\mathfrak{n}(\mathfrak{h})$ given by Eq. (3.3). The connected component of the intersection of $\mathbf{P}_{\mathfrak{h}}$ with $O_{\mathrm{p}_{0}}$ containing $\mathrm{p}_{0}$ can be described in terms of $\mathrm{N}_{0}(\mathrm{H})$ as follows,

$$
\text { component }\left(\mathrm{P}_{\mathfrak{h}} \cap O_{\mathrm{p}_{0}}\right)=\left\{\phi \mathrm{p}_{0} \mid \phi \in \mathrm{N}_{0}(\mathrm{H})\right\} \text {. }
$$


The tangent space to $\mathrm{P}_{\mathfrak{h}} \cap O_{\mathrm{p}_{0}}$ at $\mathrm{p}_{0}$ is given by

$$
\mathrm{T}_{\mathrm{p}_{0}}\left(\mathrm{P}_{\mathfrak{h}} \cap O_{\mathrm{p}_{0}}\right)=\left\{\xi_{\mathrm{p}}\left(\mathrm{p}_{0}\right) \mid \xi \in \mathfrak{n}(\mathfrak{h})\right\}
$$

For every $\xi \in \mathfrak{n}(\mathfrak{h})$ and every $w \in \mathrm{T}_{\mathrm{p}_{0}} \mathbf{M}_{\mathfrak{h}}=\mathrm{T}_{\mathrm{p}_{0}}\left(\mathbf{P}_{\mathfrak{h}} \cap \mathrm{J}-1(0)\right)$,

$$
\mu_{\mathfrak{h}}\left(\xi_{\mathbf{p}}\left(\mathrm{p}_{0}\right), w\right)=\omega\left(\xi_{\mathrm{P}}\left(\mathrm{p}_{0}\right), \mathbf{w}\right)=\left\langle\mathrm{dJ}_{\mathrm{p}_{0}}(\mathrm{w}) \mid \xi\right\rangle=0
$$

This implies that $\mathrm{T}_{\mathbf{p}_{0}}\left(\mathbf{P}_{\mathfrak{h}} \cap O_{\mathbf{p}_{0}}\right)$ is contained in the null space of $\mu_{\mathfrak{h}}$ at $\mathbf{p}_{0}$.

Let $S_{\mathrm{p}_{0}}$ be a slice through $\mathrm{p}_{0}$ satisfying Eq. (3.8). Then, by the Slice Theorem,

$$
\mathrm{T}_{\mathrm{p}_{0}} \mathbf{M}_{\mathfrak{h}}=\mathrm{T}_{\mathrm{p}_{0}}\left(\mathbf{M}_{\mathfrak{h}} \cap O_{\mathrm{p}_{0}}\right) \oplus \mathrm{T}_{\mathrm{p}_{0}}\left(\mathbf{M}_{\mathfrak{h}} \cap S_{\mathrm{p}_{0}}\right)=\mathrm{T}_{\mathrm{p}_{0}}\left(\mathrm{P}_{\mathfrak{h}} \cap O_{\mathrm{p}_{0}}\right) \oplus \mathrm{T}_{\mathrm{p}_{0}}\left(\mathbf{M}_{\mathfrak{h}} \cap S_{\mathrm{p}_{0}}\right),
$$
since $O_{\mathrm{p}_{0}} \subseteq \mathrm{J}-1(0)$. By Proposition 3.5, $\mathrm{T}_{\mathrm{p}_{0}}\left(\mathrm{M}_{\mathfrak{h}} \cap S_{\mathrm{p}_{0}}\right)$ is symplectic. Hence, $\left\{\zeta_{\mathbf{P}}\left(\mathrm{p}_{0}\right) \mid \zeta \in \mathfrak{n}(\mathfrak{h})\right\}=\mathrm{T}_{\mathrm{p}_{0}}\left(\mathbf{P}_{\mathfrak{h}} \cap O_{\mathrm{p}_{0}}\right)$ is the null space of $\mu_{\mathfrak{h}}$ at $\mathrm{p}_{0}$. This completes the proof of Theorem 3.

\section{Reduction.}

The reduced phase space $\check{\mathrm{P}}$ of the system is defined as the space of $G S(\mathrm{P})$ orbits in the constraint set $\mathrm{J}-1(0)$,

$$
\check{\mathbf{P}}=\mathrm{J}^{-1}(0) / G S(\mathrm{P}) .
$$

We denote by $\rho: \mathrm{J}^{-1}(0) \rightarrow \check{\mathrm{P}}$ the natural projection, assigning to each $\mathrm{p} \in \mathrm{J}^{-1}(0)$ the orbit $O_{\mathrm{p}} \in \check{\mathbf{P}}$

$$
\rho(\mathbf{p})=O_{\mathbf{p}}
$$

Since the action of $G S(\mathbf{P})$ in $\mathbf{P}$ is proper, the quotient topology in $\check{\mathbf{P}}$ is Hausdorff. This can be seen as follows. If $\mathbf{p}, \mathbf{q} \in \mathrm{J}^{-1}(0)$ are such that $\rho(\mathrm{p})$ and $\rho(\mathbf{q})$ cannot be separated by open sets; then there exists a sequence $\mathbf{p}_{\mathrm{n}}$ in $\mathrm{J}^{-1}(0)$ such that $\rho\left(\mathrm{p}_{\mathrm{n}}\right)$ converges both to $\rho(\mathbf{p})$ and $\rho(\mathbf{q})$. Let $S_{\mathbf{p}}$ and $S_{\mathbf{q}}$ be slices through $\mathbf{p}$ and $\mathbf{q}$, respectively. For sufficiently large $\mathrm{n}$, there exist $\phi_{\mathrm{n}}, \psi_{\mathrm{n}} \in G S(\mathrm{P})$ such that $\phi_{\mathrm{n}} \mathrm{p}_{\mathrm{n}} \in S_{\mathrm{p}}$ and $\psi_{\mathrm{n}} \mathrm{p}_{\mathrm{n}} \in S_{\mathrm{q}}$. Hence, $\phi_{\mathrm{n}} \mathrm{p}_{\mathrm{n}} \rightarrow \mathrm{p}$ and $\psi_{\mathrm{n}} \mathrm{p}_{\mathrm{n}} \rightarrow \mathrm{q}$ as $\mathrm{n} \rightarrow \infty$. Thus, $\phi_{\mathrm{n}} \psi_{\mathrm{n}}^{1}\left(\psi_{\mathrm{n}} \mathrm{p}_{\mathrm{n}}\right) \rightarrow \mathrm{p}$, while $\psi_{\mathrm{n}} \mathrm{p}_{\mathrm{n}} \rightarrow \mathrm{q}$, which implies that $\phi_{\mathrm{n}} \psi_{\mathrm{n}}^{1}$ has a convergent subsequence with limit $\chi$ and $\chi \mathbf{q}=\mathbf{p}$. Hence, $\mathbf{p} \in O_{\mathbf{q}}$ and $\rho(\mathbf{p})=\rho(\mathbf{q})$. 
If $\mathrm{H}_{1}$ and $\mathrm{H}_{2}$ are conjugate compact subgroups of $G S(\mathrm{P})$, with Lie algebras $\mathfrak{h}_{1}$ and $\mathfrak{h}_{2}$, respectively, then the $G S(\mathbf{P})$-orbits of $\mathbf{P}_{\mathfrak{h}_{1}}$ and $\mathbf{P}_{\mathfrak{h}_{2}}$ coincide, that is $\mathbf{P}_{\left(\mathfrak{h}_{1}\right)}=\mathbf{P}_{\left(\mathfrak{h}_{2}\right)}$, c.f. Eq. (3.11), and $\rho\left(\mathbf{P}_{\mathfrak{h}_{1}}\right)=\rho\left(\mathbf{P}_{\mathfrak{h}_{2}}\right)$. For every Lie algebra $\mathfrak{h}$ of a compact subgroup $\mathrm{H}$ of $G S(\mathbf{P})$, we denote by $\check{\mathbf{P}}_{(\mathfrak{h})}$ the projection of $\mathrm{J}^{-1}(0) \cap \mathbf{P}_{(\mathfrak{h})}$ to $\check{\mathbf{P}}$,

$$
\check{\mathbf{P}}_{(\mathfrak{h})}=\rho\left(J^{-1}(0) \cap \mathbf{P}_{(\mathfrak{h})}\right)
$$

and by $\rho_{\mathfrak{h}}: \mathbf{M}_{\mathfrak{h}} \rightarrow \check{\mathbf{P}}_{(\mathfrak{h})}$ the restriction of $\rho$ to $\mathbf{M}_{\mathfrak{h}}=J^{-1}(0) \cap \mathbf{P}_{\mathfrak{h}}$, considered as a map to $\check{\mathbf{P}}_{(\mathfrak{h})}$.

\section{THEOREM 4.}

$\check{\mathrm{P}}_{(\mathfrak{h})}$ is a weakly symplectic manifold with an exact symplectic form

$$
\check{\omega}_{(\mathfrak{h})}=\mathrm{d} \check{\theta}_{(\mathfrak{h})} \text {. }
$$

For every $\mathfrak{h}$ in the conjugacy class $(\mathfrak{h}), \rho_{\mathfrak{h}}: \mathrm{M}_{\mathfrak{h}} \rightarrow \check{\mathbf{P}}_{(\mathfrak{h})}$ is a submersion, and

$$
\rho_{\mathfrak{h}}^{*} \check{\theta}_{(\mathfrak{h})}=\theta \mid \mathbf{M}_{\mathfrak{h}} \text { and } \rho_{\mathfrak{h}}^{*} \check{\omega}_{(\mathfrak{h})}=\mu_{\mathfrak{h}} \text {. }
$$

The proof of this theorem will be split into several propositions.

\section{PROPOSTTION 4.1.}

Each connected component of $\check{\mathbf{P}}_{(\mathfrak{h})}$ is a smooth manifold. For each $\mathfrak{h}$ in the conjugacy class $(\mathfrak{h})$, the map $\rho_{\mathfrak{h}}: \mathbf{M}_{\mathfrak{h}} \rightarrow \check{\mathbf{P}}_{(\mathfrak{h})}$ is a submersion.

Proof. Since $\mathrm{H}$ is normal in $\mathrm{N}(\mathrm{H})$, the quotient $\mathrm{N}(\mathrm{H}) / \mathrm{H}$ is a group. We want to define an action of $\mathrm{N}(\mathrm{H}) / \mathrm{H}$ in $\mathbf{M}_{\mathfrak{h}}$. Let $\mathbf{p}$ and $\mathbf{q}=\phi p$ be two points on the same orbit of $\mathrm{N}(\mathrm{H})$ in $\mathbf{M}_{\mathfrak{h}}$. Since, $\chi \mathrm{p}=\mathrm{p}$ for every $\chi \in \mathrm{H}$, it follows that $\mathrm{q}$ is uniquely determined by $p$ and the equivalence class $[\phi]$ of $\phi$ in $N(H) / H$. Hence, we can set $[\phi] p$ $=\phi$ p. This defines a left action of $\mathrm{N}(\mathrm{H}) / \mathrm{H}$ in $\mathbf{M}_{\mathfrak{h}}$ : Moreover, $\check{\mathbf{P}}_{(\mathfrak{h})}=\rho_{\mathfrak{h}}\left(\mathbf{M}_{\mathfrak{h}}\right)$ coincides with the space of $\mathrm{N}(\mathrm{H}) / \dot{H}$ orbits in $\mathbf{M}_{\mathfrak{h}}$,

$$
\check{\mathbf{P}}_{(\mathfrak{h})}=\mathbf{M}_{\mathfrak{h}} /(\mathrm{N}(\mathrm{H}) / \mathrm{H})
$$


The $(\mathrm{N}(\mathrm{H}) / \mathrm{H})$ orbits in $\mathbf{M}_{\mathfrak{h}}$ are unions of connected components. Each $\phi \in(\mathrm{N}(\mathrm{H}) / \mathrm{H})$, which is not in $\mathrm{N}_{0}(\mathrm{H}) / \mathrm{H}$ acts as a diffeomorphism between connected components. Hence, connected components of $\check{\mathrm{P}}_{(\mathfrak{h})}$ are diffeomorphic to the quotients of connected components of $\mathbf{M}_{\mathfrak{h}}$ by $\mathrm{N}_{0}(\mathrm{H}) / \mathrm{H}$.

The map $\rho_{\mathfrak{h}}: \mathbf{M}_{\mathfrak{h}} \rightarrow \check{\mathbf{P}}_{(\mathfrak{h})}$ is onto. Furthermore, it follows from the Slice Theorem that, for each $\mathbf{p} \in \mathbf{M}_{\mathfrak{h}}$, there exists a slice $\mathbf{S}_{\mathbf{p}}$ through $\mathbf{p}$ for the action of $G S(\mathbf{P})$ in $\mathbf{P}$. By Proposition 3.5, $\mathbf{M}_{\mathfrak{h}} \cap \mathrm{S}_{\mathrm{p}}$ is a smooth manifold. It is a slice for the action of $\mathbf{N}_{0}(\mathrm{H}) / \mathrm{H}$ in $\mathbf{M}_{\mathfrak{h}}$. Then, by Eq. (4.6), there exists an open neighbourhood $\mathbf{U}_{\mathbf{p}}$ of $\mathbf{p}$ in $\mathbf{M}_{\mathfrak{h}}$ $\cap \mathbf{S}_{\mathbf{p}}$ such that $\rho_{\mathfrak{h}} \mid \dot{U}_{\mathbf{p}}$ is a homeomorphism of $U_{\mathbf{p}}$ onto $\rho_{\mathfrak{h}}\left(\mathrm{U}_{\mathbf{p}}\right) \subset \check{\mathrm{P}}_{(\mathfrak{h})}$. The family of sets $\left\{\rho_{\mathfrak{h}}\left(\mathbf{U}_{\mathbf{p}}\right) \mid \mathbf{p} \in \mathbf{M}_{\mathfrak{h}}\right\}$ is an open cover of $\check{\mathbf{P}}_{(\mathfrak{h})}$. Suppose $\mathbf{p}_{1}$ and $\mathbf{p}_{2}$ be in the same connected component of $\mathbf{M}_{\mathfrak{h}}$ and $\check{\mathbf{p}}_{0} \in \rho_{\mathfrak{h}}\left(\mathbf{U}_{\mathbf{p}_{1}}\right) \cap \rho_{\mathfrak{h}}\left(\mathbf{U}_{\mathbf{p}_{2}}\right)$. For any $\mathbf{p}_{0} \in \rho_{\mathfrak{h}}{ }^{-1}\left(\check{\mathbf{p}}_{0}\right)$ in the same connected component as $\mathbf{p}_{1}$ and $\mathbf{p}_{2}$, let $\mathrm{U}_{\mathrm{p}_{0}}$ be the chosen neighbourhood of $\mathrm{p}_{0}$ in $\mathbf{M}_{\mathfrak{h}} \cap \mathbf{S}_{\mathbf{p}_{0}}$. There exist $\phi_{1}, \phi_{2} \in \mathrm{N}_{0}(\mathrm{H})$ such that $\phi_{1} \mathbf{p}_{0} \in \mathrm{S}_{\mathbf{p}_{1}}$ and $\phi_{2} \mathbf{p}_{0} \in \mathbf{S}_{\mathbf{p}_{2}}$. Moreover, there is a map $\Phi$ from a neighbourhood $V$ of $\phi_{1} p_{0}$ in $M_{h} \cap S_{p_{1}}$ to $N_{0}(H) / H$ such that, for each $p \in V, \phi_{2} \Phi(p) \phi_{1}^{-1} p \in S_{p_{2}}$, and $\Phi\left(\phi_{1} p_{0}\right)=$ identity. Since the action of $N_{0}(H) / H$ in $M_{\mathfrak{h}}$ is locally free, for sufficiently small $V$ the map $\Phi: V \rightarrow N_{0}(H) / H$ satisfying the above conditions is unique and smooth. Hence, the map

$$
\mathrm{V} \rightarrow \mathrm{M}_{\mathfrak{h}} \cap \mathrm{S}_{\mathrm{p}_{2}}: \mathrm{p} \mapsto \phi_{2} \Phi(\mathrm{p}) \phi_{1}{ }^{-1} \mathbf{p}
$$

is a diffeomorphism onto its image. This ensures that the family maps $\left\{\left(\rho_{\mathfrak{h}} \mid \mathbf{U}_{\mathbf{p}}\right)^{-1} \mid \mathbf{p} \in \mathbf{M}_{\mathfrak{h}}\right\}$ defines a smooth atlases in connected components of $\check{\mathrm{P}}_{(\mathfrak{h})}$. In the differentiable structure defined in this way the map $\rho_{\mathfrak{h}}: \mathbf{M}_{\mathfrak{h}} \rightarrow \check{\mathbf{P}}_{(\mathfrak{h})}$ is clearly a submersion.

It remains to show that the obtained differentiable structure in $\check{\mathbf{P}}_{(\mathfrak{h})}$ is independent of the choice of $\mathfrak{h}$ in the conjugacy class $(\mathfrak{h})$. If $\mathfrak{h}_{1}$ is another 
representative of $(\mathfrak{h})$, and $\mathrm{H}_{1}$ is the group generated by it, then there exists $\phi \in G S(\mathrm{P})$ such that $\phi \mathrm{H} \phi^{-1}=\mathrm{H}_{1}$. Moreover, by Eq. (2.11), $\phi\left(\mathbf{P}_{\mathfrak{h}}\right)=\mathbf{P}_{\mathfrak{h}_{1}}$, and $\phi \mid \mathbf{P}_{\mathfrak{h}}$ is a diffeomorphism of $\mathbf{P}_{\mathfrak{h}}$ onto $\mathbf{P}_{\mathfrak{h}_{\mathfrak{l}}}$.

Q.E.D.

\section{PropostTION 4.2.}

For every $\mathfrak{h}$ in $(\mathfrak{h})$, the pull-back of the 1 -form $\theta$ to $\mathbf{M}_{\mathfrak{h}}$, denoted $\theta_{\mathfrak{h}} \mid \mathbf{M}_{\mathfrak{h}}$, is $N(H)$ invariant, and it vanishes on the vectors $\xi_{\mathbf{P}}(\mathbf{p})$, with $\mathbf{p} \in \mathbf{M}_{\mathfrak{h}}$ and $\xi \in \mathfrak{n}(\mathfrak{h})$. It pushes forward to a unique 1-form $\check{\theta}_{(\mathfrak{h})}$ on $\check{\mathrm{P}}_{(\mathfrak{h})}$ such that

$$
\rho_{\mathfrak{h}}^{*} \check{\theta}_{(\mathfrak{h})}=\theta_{\mathfrak{h}} \mid \mathbf{M}_{\mathfrak{h}} \text {. }
$$

The exterior differential $\check{\omega}_{(\mathfrak{h})}=\mathrm{d} \check{\theta}_{(\mathfrak{h})}$ is a weakly symplectic form on $\check{\mathbf{P}}_{(\mathfrak{h})}$, and

$$
\rho_{\mathfrak{h}}^{*} \check{\mathscr{\omega}}_{(\mathfrak{h})}=\mu_{\mathfrak{h}} \text {. }
$$

The forms $\check{\theta}_{(\mathfrak{h})}$ and $\check{\check{\omega}}_{(\mathfrak{h})}$ are independent of the choice of $\mathfrak{h}$ in $(\mathfrak{h})$.

PROOF. $\quad\left\langle\theta \mid \xi_{\mathrm{P}}(\mathrm{p})\right\rangle=\mathrm{J}_{\xi}(\mathrm{p})=0$ for all $\mathrm{p} \in \mathbf{M}_{\mathfrak{h}}=\mathrm{P}_{\mathfrak{h}} \cap \mathrm{J}-1(0)$ and all $\xi \in g s(\mathrm{P})$. This implies that $\theta_{\mathfrak{h}} \mid \mathbf{M}_{\mathfrak{h}}$ vanishes on $\xi_{\mathrm{p}}(\mathrm{p})$ for all $\mathrm{p} \in \mathbf{M}_{\mathfrak{h}}$ and all $\xi \in \mathfrak{n}(\mathfrak{h})$. Since $\theta$ is $G S(\mathbf{P})$ invariant, $\theta_{\mathfrak{h}} \mid \mathbf{M}_{\mathfrak{h}}$ is $\mathrm{N}(\mathrm{H})$ invariant. Hence, $\theta_{\mathfrak{h}} \mid \mathbf{M}_{\mathfrak{h}}$ pushes forward to a 1 -form $\check{\theta}_{(\mathfrak{h})}$, satisfying (4.7), which does not depend on the choice of $\mathfrak{h}$ in $(\mathfrak{h})$.

It follows from (4.7) that

$$
\rho_{\mathfrak{h}}^{*} \check{\omega}_{(\mathfrak{h})}=\rho_{\mathfrak{h}}^{*} \mathrm{~d} \check{\theta}_{(\mathfrak{h})}=\mathrm{d} \rho_{\mathfrak{h}}^{*} \check{\theta}_{(\mathfrak{h})}=\mathrm{d}\left(\theta_{\mathfrak{h}} \mid \mathbf{M}_{\mathfrak{h}}\right)=\mathrm{d} \theta_{\mathfrak{h}}\left|\mathbf{M}_{\mathfrak{h}}=\omega_{\mathfrak{h}}\right| \mathbf{M}_{\mathfrak{h}}=\mu_{\mathfrak{h}},
$$

which proves $(4.8)$. The independence of $\check{\omega}_{(\mathfrak{h})}$ from the choice of $\mathfrak{h}$ in $(\mathfrak{h})$ follows from the independence of $\check{\theta}_{(\mathfrak{h})}$ :

$$
\text { Q.E.D. }
$$

This completes the proof of Theorem 4 . 


\section{Symmetry breaking.}

Yang-Mills potentials represent connections in a right principal bundle $\mathrm{Q}$ over $\mathrm{M}$ with structure group $G$. Since $M$ is contractible, the bundle $Q$ is trivial,

$$
\mathrm{Q}=\mathrm{M} \times \mathrm{G}
$$

and the action of $G$ in $Q$ is given by

$$
\mathrm{Q} \times \mathrm{G} \rightarrow \mathrm{Q}:((\mathrm{x}, \mathrm{g}), \mathrm{h}) \mapsto((\mathrm{x}, \mathrm{g}) \cdot \mathrm{h})=(\mathrm{x}, \mathrm{gh})
$$

The associated bundle $Q[G]$ of $Q$ with typical fibre $G$ and the adjoint action of $G$ on itself is called the group bundle of $Q$. Sections of $Q[G]$ correspond to automorphisms of $\mathrm{Q}$ covering the identity transformation in $\mathrm{M}$. In this context, the group $G S(\mathrm{P})$ of gauge symmetries of $\mathbf{P}$ can be identifies with the group of sections of $Q[G]$, of class $\mathrm{H}^{3}(M)$, which satisfy the boundary condition (2.4).

Sections of associated bundles correspond to equivariant maps from the principal bundle to the typical fibre. Thus, each element $\phi \in G S(\mathrm{P})$ corresponds to a map $\phi^{\#}: \mathrm{Q} \rightarrow \mathrm{G}$ such that, for every $(\mathrm{x}, \mathrm{g}) \in \mathrm{Q}$,

$$
\phi^{\#}((x, g))=\mathrm{g}^{-1} \phi(\mathrm{x}) \mathrm{g} \text {. }
$$

The adjoint bundle of $Q$ is the associated bundle $Q[\mathfrak{g}]$ with typical fibre $\mathfrak{g}$ and the adjoint action of $\mathrm{G}$ on $\mathfrak{g}$. The space of sections of $\mathrm{Q}[\mathrm{g}]$, is the Lie algebra of the group of sections of the group bundle Q[G]. The Lie algebra $g s(\mathrm{P})$ consists of sections of the adjoint bundle, which are of Sobolev class $\mathrm{H}^{3}(\mathrm{M})$ and satisfy the boundary condition (2.1). Each $\xi: \mathbf{M} \rightarrow \mathfrak{g}$ in $g s(\mathbf{P})$ corresponds to an equivariant map $\xi^{\#}: \mathrm{P} \rightarrow \mathfrak{g}$ such that

$$
\xi^{\#}(\mathrm{x}, \mathrm{e})=\xi(\mathrm{x}) .
$$

Let $\mathfrak{h} \subset g s(\mathbf{P})$ be the symmetry algebra of $\mathrm{p}=(\mathrm{A}, \mathrm{E}, \Psi) \in \mathbf{P}$, and $\mathrm{H}$ the connected subgroup of $G S(\mathbf{P})$ with Lie algebra $\mathfrak{h}$. We have shown in Sec. 2 that $\mathrm{H}$ is compact. Let $\mathrm{x}_{0}$ be a fixed point in $\mathrm{M}$, then

$$
\mathrm{H}_{0}=\left\{\mathrm{h}\left(\mathrm{x}_{0}\right) \mid \mathrm{h} \in \mathrm{H}\right\}
$$

is a closed subgroup of $\mathrm{G}$ isomorphic to $\mathrm{H}$. Consider the set 


$$
\mathrm{Q}_{0}=\left\{(\mathrm{x}, \mathrm{g}) \in \mathrm{P}\left|\mathrm{h}^{\#}(\mathrm{x}, \mathrm{g})=\mathrm{h}\left(\mathrm{x}_{0}\right) \forall \mathrm{h}\right| \in \mathrm{H}\right\} \text {. }
$$

The right action of $k \in G$ leaves $Q_{0}$ invariant if and only if $k$ is in the centralizer $\mathrm{Z}\left[\mathrm{H}_{0}\right]$ of $\mathrm{H}_{0}$, defined by

$$
\mathrm{Z}\left[\mathrm{H}_{0}\right]=\left\{\mathrm{k} \in \mathrm{G} \mid \mathrm{kg}=\mathrm{gk} \forall \mathrm{g} \in \mathrm{H}_{0}\right\} .
$$

Actually, $\mathrm{Q}_{0}$ is a right principal bundle over $\mathbf{M}$ with structure group $\mathrm{Z}\left[\mathrm{H}_{0}\right]$. Furthermore, for each $g \in G$, the set

$$
\mathrm{H}_{\left(\mathrm{x}_{0}, \mathrm{~g}\right)}=\left\{\mathrm{h}^{\#}\left(\mathrm{x}_{0}, \mathrm{~g}\right) \mid \mathrm{h} \in \mathrm{H}\right\}=\left\{\mathrm{g}-\mathrm{l} \mathrm{h}\left(\mathrm{x}_{0}\right) \mathrm{g} \mid \mathrm{h} \in \mathrm{H}\right\}
$$

is a group conjugate to $\mathrm{H}_{0}$ with the centralizer conjugate to $\mathrm{Z}\left[\mathrm{H}_{0}\right]$. Hence, for a fixed $\mathrm{H} \subset G S(\mathrm{P})$, the principal bundle $\mathrm{Q}$ is foliated by principal sub-bundles with conjugate structure groups.

The Yang-Mills potential $\mathbf{A}$ gives a local description of a connection in $\mathrm{Q}$ relative to the trivialization given by the product structure (5.1). The corresponding connection form $\alpha$ on $Q$ is given by

$$
\alpha=\gamma^{-1} \mathrm{~A} \gamma+\gamma^{-1} \mathrm{~d} \gamma
$$

where $\gamma$ is the embedding of $G$ into the matrix group $g l\left(\mathbb{R}^{\mathrm{k}}\right)$. The horizontal distribution hor $\mathrm{TQ}$ on $\mathrm{Q}$ is the kernel of the connection form. The connection in $\mathrm{Q}$ is said to reduce to a connection in a sub-bundle $\mathrm{Q}_{0}$ if hor $\mathrm{TQ} \mid \mathrm{Q}_{0} \subset$ hor $\mathrm{TQ}_{0}$.

\section{PROPOSITION 5.1.}

Let $\mathfrak{h} \subset g s(\mathrm{P})$ be the stability algebra of $\mathrm{p}=(\mathrm{A}, \mathrm{E}, \Psi) \in \mathrm{P}$, and $\mathrm{H}$ the connected subgroup of $G S(\mathrm{P})$ with Lie algebra $\mathfrak{h}$. Then, connection in $\mathrm{Q}$ defined by $\mathrm{A}$ reduces to a connection in the sub-bundle $\mathrm{Q}_{0}$ with structure group $\mathrm{Z}\left[\mathrm{H}_{0}\right]$.

Proof. Let $q(t)$ be a horizontal curve in $Q$. For every $h \in H, \frac{d}{d t} h^{\#}(q(t))$ is the equivariant function on $Q$ describing the covariant derivative of the section $h$ of $Q[G]$ along $\mathrm{q}(\mathrm{t})$. However, $\stackrel{\mathrm{H}}{\subseteq} G S_{\mathrm{p}}$ implies that every $\mathrm{h} \in \mathrm{H}$ is covariantly constant. 
Hence, every horizontal curve through $Q_{0}$ is contained in $Q_{0}$, which means that the connection in $\mathrm{Q}$ defined by $\mathbf{A}$ reduces to a connection in $\mathrm{Q}_{0}$.

Q.E.D.

The electric component $\mathbf{E}$ of the field strength is a $\mathfrak{g}$-valued 1 -form on $\mathbf{M}$. It can be interpreted as a section of the bundle $T^{*} M \otimes Q[\mathfrak{g}]$ over $M$. Let $E^{\#}$ be the corresponding equivariant form on $Q$ with values in $\mathfrak{g}$. If $\mathfrak{h}$ is the symmetry algebra of E, then (1.6) yields

$$
[\mathrm{E}, \xi]=0 \quad \forall \quad \xi \in \mathfrak{h} .
$$

This implies that

$$
\left[\mathbf{E}^{\#}, \xi^{\#}\right]=0 \quad \forall, \xi \in \mathfrak{h} .
$$

At points of $\mathrm{Q}_{0}$, given by (5.6), we have $\xi^{\#} \mid \mathrm{Q}_{0}=\xi\left(\mathrm{x}_{0}\right)$. Hence, for each point $(\mathrm{x}, \mathrm{g}) \in$ Qo,

$$
\left[\mathrm{E}^{\#}(\mathrm{x}, \mathrm{g}), \xi\left(\mathrm{x}_{0}\right)\right]=0 \quad \forall \quad \xi \in \mathfrak{h},
$$

which implies that, for all $(x, g) \in Q_{0}, E^{\#}(x, g)$ is in the Lie algebra $\hat{z}\left[H_{0}\right]$ of the centralizer $\mathrm{Z}\left[\mathrm{H}_{0}\right]$ of $\mathrm{H}_{0}$. Hence, the electric component $\mathbf{E}$ of the field strength reduces to a section $\mathrm{E}_{0}$ of the bundle $\mathrm{T}^{*} \mathrm{Q}_{0} \otimes \mathrm{Q}\left[\hat{z}\left[\mathrm{H}_{0}\right]\right]$ over $\mathrm{M}$.

The matter field $\Psi$ is a section of the associated bundle of $Q$, with typical fibre $\mathbb{R}^{\mathrm{n}} \otimes \mathbb{R}^{4}$, where $\mathbb{R}^{\mathrm{n}}$ is the space of the fundamental representation of (the matrix group) $G$, and the factor $\mathbb{R}^{4}$ describes the spin degrees of freedom. It follows from (1.6) that $(\mathbf{A}, \mathbf{E}, \Psi) \in \mathbf{P}_{\mathfrak{h}}$ implies that

$$
\xi \Psi=0 \quad \forall \quad \xi \in \mathfrak{h} .
$$

Let $\Psi^{\#}$ be the equivariant function from $Q$ to $\mathbb{R}^{\mathrm{n}} \otimes \mathbb{R}^{4}$ corresponding to $\Psi$. Then, (5.12) yields

$$
\xi^{\#} \Psi^{\#}=0 \quad \forall \quad \xi \in \mathfrak{h}
$$

For each point $(x, g) \in \dot{\mathrm{Q}}_{0}$.

$$
\xi\left(\mathrm{x}_{0}\right) \Psi^{\#}(\mathrm{x}, \mathrm{g})=0 \quad \forall \quad \xi \in \mathfrak{h},
$$


which implies that $\Psi^{\#}(x, g)$ is in the subspace

$$
\mathrm{V}_{0}=\left\{\mathrm{z} \in \mathbb{R}^{\mathrm{n}} \otimes \mathbb{R}^{4} \mid \xi\left(\mathrm{x}_{0}\right) \mathrm{z}=0 \forall \xi \in \mathfrak{h}\right\}
$$

of $\mathbb{R}^{\mathrm{n}} \otimes \mathbb{R}^{4}$ annihilated by Lie algebra $\mathfrak{h}_{0}$ of $\mathrm{H}_{0}$. The action of the centralizer $\mathrm{Z}\left[\mathrm{H}_{0}\right]$ preserves $V_{0}$. Hence, the matter field $\Psi$ reduces to a section $\Psi_{0}$ of the associated bundle of $\mathrm{Q}_{0}$, with typical fibre $\mathrm{V}_{0} \subseteq \mathbb{R}^{\mathrm{n}} \otimes \mathbb{R}^{4}$.

Thus, we have proved

\section{THEOREM 5.}

For every $(\mathbf{A}, E, \Psi) \in \mathbf{P}_{\mathfrak{h}}$, the Cauchy data $(\mathbf{A}, \mathbf{E})$ for the Yang-Mills theory with the structure (internal symmetry)-group $G$ reduce to Cauchy data for a Yang-Mills theory with principal bundle

$$
\mathrm{Q}_{0}=\left\{(\mathrm{x}, \mathrm{g}) \in \mathrm{P} \mid \mathrm{h}^{\#}(\mathrm{x}, \mathrm{g})=\mathrm{h}\left(\mathrm{x}_{0}\right) \forall \mathrm{h} \in \mathrm{H}\right\}
$$

and structure (internal symmetry) group

$$
\mathrm{Z}\left[\mathrm{H}_{0}\right]=\left\{\mathrm{g} \in \mathrm{G} \mid \mathrm{gh}\left(\mathrm{x}_{0}\right)=\mathrm{h}\left(\mathrm{x}_{0}\right) \mathrm{g} \forall \mathrm{h} \in \mathrm{H}\right\},
$$

where $x_{0}$ is and arbitrary fixed point of $M$, and the matter field $\Psi$ reduces to a section of the associated bundle of $\mathrm{Q}_{0}$ with typical fibre

$$
\mathrm{V}_{0}=\left\{\mathrm{z} \in \mathbb{R}^{\mathrm{n}} \otimes \mathbb{R}^{4}\left|\xi\left(\mathrm{x}_{0}\right) \mathrm{z}=0 \forall \xi \in\right| \mathfrak{h}\right\} \text {. }
$$

The change of the base point $x_{0}$ correspond to passing from $Q_{0}$ to another principal sub-bundle of $\mathrm{Q}$ with conjugate structure group.

It follows from Theorem 5 that each symplectic manifold $\check{\mathbf{P}}_{(\mathfrak{h})}$ in $\check{\mathbf{P}}$ corresponds to symmetry breaking from the original internal symmetry group $G$ to conjugacy classes of subgroups $\mathrm{Z}\left[\mathrm{H}_{0}\right]$ centralizing $\mathrm{H}_{0}$. It should be noted that the symmetry breaking encountered here is completely intrinsic, it does not require additional Higgs fields. On the other hand, it does not lead to vector bosons. 
Appendix A. Completion and almost complex structure.

One of the technical assumptions in [3] is the existence of an appropriate almost complex structure, which in Yang-Mills theory acts by interchanging $\mathbf{A}$ and $\mathbf{E}$. However, in our phase space $\mathbf{P}$ the variables $\mathbf{A}$ and $\mathbf{E}$ appear asymmetrically, and we do not have existence and uniqueness theorems in spaces symmetric under the interchange of $\mathbf{A}$ and E.

Let $\tilde{\mathbf{P}}$ denote the completion of $\mathbf{P}$ in the $\mathrm{L}^{2}$ norm. The weak symplectic form $\omega$ in $\mathbf{P}$ induces a strong symplectic form $\tilde{\omega}$ in $\tilde{\mathbf{P}}$. The $\mathrm{L}^{2}$ scalar product $\langle. \mid .\rangle_{\mathrm{L}^{2}}$ defines a Riemannian metric in $\tilde{\mathbf{P}}$. Let $\mathcal{J}: \mathrm{T} \tilde{\mathbf{P}} \rightarrow \mathrm{T} \tilde{\mathbf{P}}$ be defined by

$$
J(\delta \mathrm{A}, \delta \mathrm{E}, \delta \Psi)=(-\delta \mathrm{E}, \delta \mathrm{A}, i \Psi)
$$

for every $(\delta \mathrm{A}, \delta \mathrm{E}, \delta \Psi) \in \mathrm{T} \tilde{\mathbf{P}}$. Then, $\mathcal{F}=-1$, and

$$
\tilde{\omega}(\mathcal{J u}, \mathcal{J} v)=\tilde{\omega}(\mathrm{u}, \mathrm{v})=\langle\mathcal{J} u \mid v\rangle_{\mathrm{L}^{2}}=-\langle\mathrm{u} \mid \mathcal{J} \mathrm{v}\rangle_{\mathrm{L}^{2}}
$$

for all $u, v \in T \tilde{P}$. Thus, $\mathcal{J}$ is an almost complex structure on $\tilde{\mathbf{P}}$. The action of $G S(\mathbf{P})$ in $\mathbf{P}$ extends to an action in $\tilde{\mathbf{P}}$ preserving its symplectic form, the Riemannian metric and the almost complex structure.

Let $V$ be a closed subspace of $T_{p} P$ and let $\tilde{V}$ be its closure in $T_{p} \tilde{P}$. The symplectic annihilator $\mathrm{V}^{\omega}$ of $\mathrm{V}$ is defined by

$$
\mathbf{V}^{(\omega)}=\left\{\mathbf{u} \in \mathrm{T}_{\mathbf{p}} \mathbf{P} \mid \omega(\mathbf{u}, \mathbf{v})=0 \forall \mathbf{v} \in \mathbf{V}\right\}
$$

Similarly, the symplectic annihilator of $\tilde{V}$ in $T_{p} \tilde{P}$ is

$$
\tilde{\mathbf{V}}^{\tilde{\omega}}=\left\{\mathbf{u} \in \mathrm{T}_{\mathbf{P}} \tilde{\mathbf{P}} \mid \tilde{\omega}(\mathbf{u}, \mathbf{v})=0 \forall \mathbf{v} \in \tilde{\mathbf{V}}\right\}
$$

Since $\mathbf{V}$ is closed, we have

$$
\left(V^{a j}\right)^{\omega}=\mathrm{V}
$$

We denote by $\mathrm{V}^{\perp}$ the $\mathrm{L}^{2}$-orthogonal complement of $\mathrm{V}$ in $\mathrm{T}_{\mathbf{P}} \mathbf{P}$, and $\tilde{\mathrm{V}}^{\perp}$ the $\mathrm{L}^{2}$ orthogonal complement of its closure $\tilde{V}$ in $T_{\mathbf{p}} \tilde{\mathbf{P}}$. We have

Moreover, by Eq. (A.2)

$$
\left(\mathbf{V}^{\perp}\right)^{\omega}=\left(\tilde{\mathbf{V}}^{\perp}\right)^{\tilde{\omega}} \cap \mathrm{T}_{\mathbf{p}} \mathbf{P}
$$




$$
\left(\tilde{\mathbf{V}}^{\perp}\right)^{\tilde{\omega}}=\left\{\mathbf{u} \in \mathrm{T}_{\mathbf{p}} \tilde{\mathbf{P}} \mid \tilde{\omega}(\mathbf{u}, \mathbf{v})=0 \forall \mathbf{v} \in \tilde{\mathbf{V}}^{\perp}\right\}=\left\{\mathbf{u} \in \mathrm{T}_{\mathbf{p}} \tilde{\mathbf{P}} \mid \mathcal{J} \mathbf{u} \in\left(\tilde{\mathbf{V}}^{\perp}\right)^{\perp}\right\}=\mathcal{J} \tilde{\mathbf{V}} .
$$

Hence,

$$
\left(\mathbf{V}^{\perp}\right)^{\omega}=\tilde{j} \tilde{\mathbf{V}} \cap \mathrm{T}_{\mathbf{p}} \mathbf{P}
$$

In the following we shall use the notation

$$
\mathcal{J}=\mathcal{J} \tilde{\mathbf{V}} \cap \mathrm{T}_{\mathbf{p}} \dot{P}
$$

Since $\mathcal{J}^{2}=-\mathrm{I}$, we have

$$
\mathcal{J} \mathbf{V}=\mathbf{V}, \text { and }(\mathcal{J} \mathbf{V}) \cap \mathbf{V}=\{0\}
$$

For each $\mathbf{p} \in \mathbf{P}$, the second derivative of the momentum map $\mathrm{J}: \mathbf{P} \rightarrow g s(\mathbf{P}){ }^{*}$ is a symmetric bilinear map $\mathrm{D}^{2 \mathrm{~J}} \mathbf{p}: \mathrm{T}_{\mathbf{p}} \mathbf{P} \times \mathrm{T}_{\mathbf{p}} \mathbf{P} \rightarrow g s(\mathbf{P}){ }^{*}$. It extends to a symmetric bilinear $\operatorname{map} \mathrm{D}^{2} \tilde{\mathrm{J}}_{\mathbf{P}}: \mathrm{T}_{\mathbf{P}} \tilde{\mathbf{P}} \times \mathrm{T}_{\mathbf{p}} \tilde{\mathbf{P}} \rightarrow g s(\mathbf{P})^{*}$.

\section{LEMma A.1.}

For all $\mathbf{v}, \mathbf{w} \in \mathrm{T}_{\mathbf{p}} \mathbf{P}$, and all $\xi \in g s(\mathbf{P})$,

$$
\left\langle D^{2} \tilde{J}_{\mathbf{p}}(\mathcal{J} \mathbf{v}, \mathcal{J} \mathbf{w}) \mid \xi\right\rangle=\left\langle D^{2} \mathrm{~J}_{\mathbf{p}}(\mathbf{v}, \mathbf{w}) \mid \xi\right\rangle \text {. }
$$

Proof. For $\xi \in g s(\mathrm{P})$, and a constant vector field $\mathbf{X}$ in $\mathbf{P}$, with $\mathbf{X}(\mathbf{p})=\mathbf{w}$, the equation $\tilde{\omega}\left(\xi_{\tilde{\mathbf{P}}}, \mathbf{X}\right)=\langle\mathrm{dJ}(\mathbf{X}) \mid \xi\rangle$, differentiated at $\mathbf{p}$ in the direction $\mathbf{v} \in \mathrm{T}_{\mathbf{P}} \mathbf{P}$ yields

$$
\tilde{\omega}\left(\mathrm{D}, \xi_{\tilde{\mathbf{P}}}(\mathbf{p}) \mathbf{v}, \mathbf{w}\right)=\left\langle\mathrm{D}^{2} \mathrm{~J} \mathbf{p}(\mathbf{v}, \mathbf{w}) \mid \xi\right\rangle \text {. }
$$

Since the action of $G S(\mathbf{P})$ in $\tilde{\mathbf{P}}$ preserves the almost complex structure $\mathcal{J}$, for every $\xi \in$ $g s(\mathrm{P})$, we have $J \circ T \xi_{\tilde{\mathbf{P}}}=\mathrm{T} \xi_{\tilde{\mathbf{P}}} \circ \mathcal{J}$. Hence,

$$
\left\langle\mathrm{D}^{2 J} \mathrm{p}_{\mathbf{p}}(\mathcal{J} \mathbf{v}, \mathcal{J} \mathbf{w}) \mid \xi\right\rangle=\tilde{\omega}\left(\mathrm{D} \xi_{\tilde{\mathbf{p}}}(\mathrm{p}) \mathcal{J} \mathbf{v}, \mathcal{J} \mathbf{w}\right)=\tilde{\omega}\left(\mathrm{d} \xi_{\tilde{\mathbf{p}}}(\mathrm{p}) \mathbf{v} ; \mathbf{w}\right)=\left\langle\mathrm{D}^{2} \mathrm{~J}_{\mathbf{p}}(\mathbf{v}, \mathbf{w}) \mid \xi\right\rangle
$$

Q.E.D.

Appendix B. Properness of the action of the gauge symmetry group.

The gauge symmetry group GS(P) consists of map $\phi: \mathrm{M} \rightarrow \mathrm{G}$ in the Sobolev class $\mathrm{H}^{3}(\mathrm{M})$ such that $n \cdot \operatorname{grad} \phi=0,(2.4)$. Its action in $\mathbf{P}$ is given by (1.5). In order 
to prove that this action is proper, we need to show that, for every sequence $\mathbf{p}_{n}=\left(\mathbf{A}_{n}, \mathbf{E}_{n}, \Psi_{n}\right)$ converging to $\mathbf{p}_{\infty}=\left(\mathbf{A}_{\infty}, \mathbf{E}_{\infty}, \Psi_{\infty}\right) \in \mathbf{P}$, and every sequence $\phi_{n}$ in $G S(P)$ such that $\phi_{\mathrm{n}} \mathrm{p}_{\mathrm{n}}$ converges to $\mathrm{p}=(\mathrm{A}, \mathrm{E}, \Psi)$, the sequence $\phi_{\mathrm{n}}$ has a convergent subsequence with limit $\phi$ and $\phi \mathbf{p}_{\infty}=\mathbf{p}$.

The gauge transformations act on $\mathbf{A}, \mathbf{E}$, and $\Psi$ independently. Hence, we may consider first the action of $G S(\mathrm{P})$ on the connections. For a sequence $\mathrm{A}_{\mathrm{n}}$ converging to $\mathrm{A}_{\infty}$, and a sequence $\phi_{\mathrm{n}}$ in $G S(\mathrm{P})$, let

$$
\mathbf{C}_{\mathrm{n}}=\phi_{\mathrm{n}} \mathbf{A}_{\mathrm{n}} \phi_{\mathrm{n}}^{-1}+\phi_{\mathrm{n}} \mathrm{d} \phi_{\mathrm{n}}^{-1}
$$

denote $A_{n}$ transformed by $\phi_{n}$. This implies

$$
\mathrm{d} \phi_{\mathrm{n}}=\phi_{\mathrm{n}} \mathbf{A}_{\mathrm{n}}-\mathbf{C}_{\mathrm{n}} \phi_{\mathrm{n}}
$$

By hypothesis, the sequences $A_{n}$ and $C_{n}$ converge in $H^{2}(M)$ to $A_{\infty}$ and $A$, respectively. In particular, their $\mathrm{H}^{2}(\mathrm{M})$ norms $\left\|\mathrm{A}_{\mathrm{n}}\right\|_{\mathrm{H}^{2}}$ and $\left\|\mathrm{C}_{\mathrm{n}}\right\|_{\mathrm{H}^{2}}$ are bounded. Furthermore, the $\mathrm{L}^{2}(\mathrm{M})$ norms $\left\|\phi_{\mathrm{n}}\right\|_{\mathrm{L}^{2}}$ of $\phi_{\mathrm{n}}$ are bounded since $\mathrm{M}$ and $\mathrm{G}$ are compact. Eq. (B.2) implies that also the $\mathrm{L}^{2}(\mathrm{M})$ norms $\left\|\mathrm{d} \phi_{\mathrm{n}}\right\|_{\mathrm{L}^{2}}$ of $\mathrm{d} \phi_{\mathrm{n}}$ are bounded. Hence, the $\mathrm{H}^{1}(\mathrm{M})$ norms $\left\|\phi_{\mathrm{n}}\right\|_{\mathrm{H}^{1}}$ of $\phi_{\mathrm{n}}$ are bounded. Repeating this argument twice, we conclude that the $\mathrm{H}^{3}(\mathrm{M})$ norms of $\phi_{\mathrm{n}}$ are bounded. By Rellich's Lemma the sequence $\dot{\phi}_{\mathrm{n}}$ has a subsequence convergent to $\phi$. in $\mathrm{H}^{2}(\mathrm{M})$. Without loss of generality, we can restrict our argument to this subsequence, and assume that $\phi_{\mathrm{n}}$ converges to $\phi$ in $\mathrm{H}^{2}(\mathrm{M})$. Hence, the sequence $\mathrm{C}_{\mathrm{n}}=\phi_{\mathrm{n}} \mathrm{A}_{\mathrm{n}} \phi_{\mathrm{n}}^{-1}+$ $\phi_{\mathrm{n}} \mathrm{d} \phi_{\mathrm{n}}^{-1}$ converges to $\phi \mathrm{A}_{\infty} \phi^{-1}+\phi \mathrm{d} \phi^{-1}$ in $\mathrm{H}^{1}(\mathrm{M})$,

$$
\left\|\phi \mathrm{A}_{\infty} \phi^{-1}+\phi \mathrm{d} \phi^{-1}-\mathbf{C}_{\mathrm{n}}\right\|_{\mathrm{H}^{1}} \rightarrow 0 \text { as } \mathrm{n} \rightarrow \infty \text {. }
$$

By hypothesis, $C_{n}$ converges to $A$ in $H^{2}(M)$. Hence,

$$
\left\|\phi \mathbf{A}_{\infty} \phi^{-1}+\phi \mathrm{d} \phi^{-1}-\mathbf{A}\right\|_{\mathrm{H}^{1}} \leq\left\|\phi \mathbf{A}_{\infty} \phi^{-1}+\phi \mathrm{d} \phi^{-1}-\mathbf{C}_{\mathrm{n}}\right\|_{\mathrm{H}^{1}}+\left\|\mathbf{C}_{\mathrm{n}}-\mathbf{A}\right\|_{\mathrm{H}^{1}} \rightarrow 0 \text { as } \mathrm{n} \rightarrow \infty \text {. }
$$

This implies that

$$
\mathbf{A}=\phi \mathbf{A}_{\infty} \dot{\phi}^{-1}+\phi \mathrm{d} \phi^{-1}
$$

and hence,

$$
\mathrm{d} \phi=\phi \mathrm{A}_{\infty}-\mathbf{A} \phi .
$$


Since the right hand side of (B.5) belongs to $\mathrm{H}^{2}(\mathrm{M})$, it follows that $\mathrm{d} \phi \in \mathrm{H}^{2}(\mathrm{M})$, so that $\phi \in \mathrm{H}^{3}(\mathrm{M})$.

Using (B.2) and (B.5), we observe that

$$
\begin{gathered}
\left\|\mathrm{d} \phi_{\mathrm{n}}-\mathrm{d} \phi\right\|_{\mathrm{H}^{2}}=\left\|\phi_{\mathrm{n}} \mathrm{A}_{\mathrm{n}}-\mathrm{C}_{\mathrm{n}} \phi_{\mathrm{n}}-\left(\phi \mathrm{A}_{\infty}-\mathbf{A} \phi\right)\right\|_{\mathrm{H}^{2}} \leq \\
\left.\left\|\phi_{\mathrm{n}} \mathbf{A}_{\mathrm{n}}-\phi \mathrm{A}_{\infty}\right\|_{\mathrm{H}^{2}}+\| \mathrm{C}_{\mathrm{n}} \phi_{\mathrm{n}}-\mathbf{A} \phi\right) \|_{\mathrm{H}^{2}} .
\end{gathered}
$$

As $\mathbf{n} \rightarrow \infty$ the right hand side tends to zero, because $\phi_{n} \rightarrow \phi, \mathbf{A}_{\mathbf{n}} \rightarrow \mathbf{A}_{\infty}$, and $\mathbf{C}_{\mathbf{n}} \rightarrow \mathbf{A}$ in $\mathrm{H}^{2}(\mathrm{M})$. Hence, $\left\|\mathrm{d} \phi_{\mathrm{n}}-\mathrm{d} \phi\right\|_{\mathrm{H}^{2}} \rightarrow 0$, which implies that $\phi_{\mathrm{n}} \rightarrow \phi$ in $\mathrm{H}^{3}(\mathrm{M})$. This proves the properness of the action of $G S(\mathrm{P})$ on the space of $\mathrm{H}^{2}(\mathrm{M})$ connections satisfying the boundary conditions (1.1).

In remains to show that $\phi$ takes $\mathrm{E}_{\infty}$ to $\mathbf{E}$ and $\Psi_{\infty}$ to $\Psi$. By hypothesis $\mathrm{E}_{\mathrm{n}} \rightarrow \mathrm{E}_{\infty}$ and $\phi_{n} E_{n} \phi_{n}^{-1} \rightarrow E$ in $H^{1}(M)$. Since $\phi_{n} \rightarrow \phi$ in $H^{3}(M)$, and a pointwise multiplication of functions in $\mathrm{H}^{1}(\mathrm{M})$ by functions in $\mathrm{H}^{3}(\mathrm{M})$ is a continuous map from $\mathrm{H}^{1}(\mathrm{M}) \times \mathrm{H}^{3}(\mathrm{M})$ to $\mathrm{H}^{1}(\mathrm{M})$, we obtain

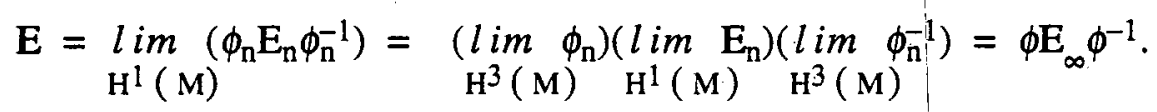

In a similar manner we obtain

$$
\Psi=\lim _{H^{2}(M)}\left(\phi_{n} \Psi_{n}\right)=\lim _{H^{3}(M)}\left(\phi_{n}\right) \lim _{H^{2}(M)}\left(\Psi_{n}\right)=\phi \Psi_{\infty} .
$$

This completes the proof of properness of the action of $G S(\mathrm{P})$ in $\mathrm{P}$.

Appendix C. Proof of the slice theorem.

We establish here the slice theorem for infinite dimensional groups, c.f. [14]. Since the assumptions made here are more general than in the body of the paper, we use an independent notation following that of Appendix 2 of [15].

Let $M$ be a Hilbert manifold, and $G$ a Hilbert Lie group, with a continuous proper smooth left action $\Phi: G \times M \rightarrow M$. In the following we use the notation $\Phi_{g}(\mathrm{~m})$ $=\Phi(g, m)$. Let $g$ be the Lie algebra of $G$. For each $m \in M, \mid$ we denote by $G_{m}$ the isotropy group of $\mathrm{m}$, by $g_{\mathrm{m}}$ the Lie algebra. of $\mathrm{G}_{\mathrm{m}}$, and by $O_{\mathrm{m}}=\mathrm{G} \cdot \mathrm{m}$ the 
orbit of $\mathrm{G}$ through $\mathrm{m}$. Since the action is proper $\mathrm{G}_{\mathrm{m}}$ is compact and the orbit $O_{\mathrm{m}}$ is closed. The tangent space $\mathrm{T}_{\mathrm{m}} O_{\mathrm{m}}$ can be presented as $\mathfrak{g} \cdot \mathrm{m}=\mathrm{T} \Phi(\mathfrak{g}, 0)(e, \mathrm{~m})$, and $\mathfrak{g}_{\mathrm{m}} \cdot \mathrm{m}=$ 0.

\section{HYPOTHESES:}

(a) The group $G$ is a Lie group in the sense that the exponential map gives a diffeomorphism of a neighbourhood of $0 \in \mathfrak{g}$ onto a neighbourhood of $e \in \mathrm{G}$.

(b) The action $\Phi$ is proper.

(c). Bochner Linearization Lemma, [16]. There is a $G_{m}$ invariant neighbourhood $\mathrm{U}$ of $\mathrm{m} \in \mathrm{M}$ and a diffeomorphism $\psi: \mathrm{U} \rightarrow \mathrm{T}_{\mathrm{m}} \mathrm{M}$ such that:

$$
\psi(\mathrm{m})=0 \text { and } \mathrm{T}_{\mathrm{m}} \psi=\text { identity }
$$

and, for every $g \in \mathrm{G}_{\mathrm{m}}$ and $\mathrm{p} \in \mathrm{U}$

$$
\psi(\Phi(\mathrm{p}))=\mathrm{T}_{\mathrm{m}} \Phi(\psi(\mathrm{p}))
$$

These assumptions are stronger than needed to get slices, but they allow us to control the topology of the space of orbits of the group action. They |are satisfied by the gauge symmetry group. $G S(\mathbf{P})$ considered in this paper. Proposition 2.1 guarantees assumption (a). Properness of the action of $G S(\mathbf{P})$ is proved in Appendix B. The Bochner Linearization Lemma follows from the fact that the action of $G S(\mathrm{P})$ is affine.

First we need a lemma.

\section{Lemma C.1.}

Given $m \in M$, let $L$ be a submanifold of $G$ through $e$ such that

$$
\mathfrak{g}=\mathfrak{g}_{\mathrm{m}} \oplus \mathrm{T}_{\mathrm{e}} \mathrm{L},
$$

and let $S$ be a submanifold of $M$ through $m$ such that

$$
\mathrm{T}_{\mathrm{m}} \mathrm{M}=\mathrm{T}_{\mathrm{m}} O_{\mathrm{m}} \oplus \mathrm{T}_{\mathrm{m}} \mathrm{S} .
$$


Then there is an open set $U \times V \subseteq L \times S$ such that $\Phi \mid(U \times V)$ is a diffeomorphism onto an open neighbourhood $\mathrm{W}$ of $\mathrm{m} \in \mathbf{M}$.

Proof. Let D $\Phi: T G \times T M \rightarrow$ TM denote the derivative of $\Phi$, and $D_{i} \Phi$ be the restriction of $\mathrm{D} \Phi$ to the $\mathrm{i}^{\prime}$ th factor. Since $\Phi(e, \mathrm{~m})=\mathrm{m}$ for all $\mathrm{m} \in \mathrm{M}$, we have that $\mathrm{D}_{2} \Phi_{(e, \mathrm{~m})}=$ identity, and so $\mathrm{D} \Phi_{(e, \mathrm{~m})}$ is surjective. Now $\operatorname{ker} \mathrm{D}_{1} \dot{\Phi}_{(e, \mathrm{~m})}=\mathfrak{g}_{\mathrm{m}}$ by definition, and also, by definition image $\mathrm{D}_{1} \Phi_{(e, \mathrm{~m})}=\mathrm{T}_{\mathrm{m}} O_{\mathrm{m}}$.

Choosing $\mathrm{L}$ and $\mathrm{S}$ so that we can make the identifications

$$
\begin{gathered}
\mathrm{T}_{e} \mathrm{~L} \cong g / g_{\mathrm{m}} \\
\mathrm{T}_{\mathrm{m}} \mathrm{S} \cong \mathrm{T}_{\mathrm{m}} \mathrm{M} / \mathrm{T}_{\mathrm{m}} O_{\mathrm{m}}
\end{gathered}
$$

we have that $D \Phi \mid\left(T_{e} L \times T_{m} S\right)$ is an isomorphism. Since $M$ is a Hilbert manifold the Lemma now follows by the inverse function theorem.

\section{Corollary C.2.}

If $\Phi_{g} \mathrm{~V} \cap \mathrm{V} \neq \varnothing$ for some $g \in \mathrm{U} \subseteq \mathrm{L} \subset \mathrm{G}$, and $\mathrm{V} \subseteq \mathrm{S}$, then $g=e$.

ProOF. Let $\mathrm{m} \in \mathrm{V}$ be such that $\Phi(g, \mathrm{~m})=\Phi\left(e, \mathrm{~m}^{\prime}\right)$ with $\mathrm{m}^{\prime} \in \mathrm{V}$. Since $\Phi$ is a local diffeomorphism on $\mathrm{U} \times \mathrm{V}$ it follows that $(g, \mathrm{~m})=\left(e, \mathrm{~m}^{\prime}\right)$, so that $g=e$.

\section{Q.E.D.}

\section{LEMMA C.3.}

For every neighbourhood $\tilde{U}$ on $M$ containing $m$, there is a $G_{m}$ invariant open set $\mathrm{U}$ containing $\mathrm{m}$ with $\mathrm{U} \subseteq \tilde{\mathrm{U}}$.

Proof. Since $M$ is a Hilbert manifold, it is first countable. Hence, there exists a sequence $\left\{U_{n}\right\}$ of neighbourhoods of $m$ in $M$ such that $U_{n} \subseteq U_{n-1}, \bigcap_{n=1}^{\infty} U_{n}=\{m\}$, and 
$\mathrm{G}_{\mathrm{m}} \cdot \mathrm{U}_{\mathrm{n}}$ is not contained in $\tilde{\mathrm{U}}$. Suppose now that the statement of the lemma is false. Then $G_{m} \cdot U_{n}$ is not contained in $\tilde{U}$ for all $n$. Hence, there exist sequences $m_{n} \in U_{n}$ and $g_{\mathrm{n}} \in \mathrm{G}_{\mathrm{m}}$ such that $g_{\mathrm{n}} \mathrm{m}_{\mathrm{n}} \notin \tilde{U}$. Since the action of $\mathrm{G}$ is proper, the isotropy group $G_{m}$ is compact and the sequence $g_{\mathrm{n}}$ has a convergent subsequence. Without loss of generality we may assume that $g_{\mathrm{n}}$ converges to $g \in \mathrm{G}_{\mathrm{m}}$. The sequence $\mathrm{m}_{\mathrm{n}}$ converges to m by construction. The continuity of the action of $G$ in $M$ implies that $g_{n} m_{n}$ converges to $g \cdot m=m$, which contradicts the statement that $g_{n} m_{n} \notin \tilde{U}$ for all $n$.

Q.E.D.

\section{SLICE THEOREM.}

For each $\mathrm{m} \in \mathrm{M}$, there exists a smooth submanifold $\mathrm{S}$ of $\mathrm{M}$ through $\mathrm{m}$ such that

$$
\begin{gathered}
\mathrm{T}_{\mathrm{m}} \mathrm{M}=\mathrm{T}_{\mathrm{m}} O_{\mathrm{m}} \oplus \mathrm{T}_{\mathrm{m}} \mathrm{S} \\
\mathrm{T}_{\mathrm{p}} \mathrm{M}=\mathrm{T}_{\mathrm{p}} O_{\mathrm{p}}+\mathrm{T}_{\mathrm{p}} \mathrm{S} \forall \mathrm{p} \in \mathrm{S} . \\
\mathrm{G}_{\mathrm{m}} \cdot \mathrm{S} \subseteq \mathrm{S} \\
\text { For } \mathrm{p} \in \mathrm{S} \text {, and } g \in \mathrm{G} \text {, if } \Phi_{g}(\mathrm{p}) \in \mathrm{S} \text { then } g \in \mathrm{G}_{\mathrm{m}}
\end{gathered}
$$

ProOF. We prove the existence of a slice by constructing a candidate $\mathrm{S}_{\varepsilon}$ and showing that properties (1) through (4) hold.

Observe that if $k \in \mathrm{G}_{\mathrm{m}}, \mathrm{kg} \cdot \mathrm{m}=\mathrm{kgk-1} \cdot \mathrm{m}$, or

$$
\Phi_{k} \circ \Phi_{g}(\mathrm{~m})=\Phi_{k g k^{-1}}(\mathrm{~m}) \text {. }
$$

If $g=\exp (\mathrm{t} \xi), \quad \xi \in \mathfrak{g}$, then the 1-parameter groups $\mathrm{t} \mapsto k[\exp (\mathrm{t} \xi)] k^{-1}$ and $\mathrm{t} \mapsto \exp \left(\mathrm{t} A d_{k} \xi\right)$ have the same tangent vector $A d_{k} \xi$ at $\mathrm{t}=0$. Hence, differentiating (C.11) with respect to $t$ at $t=0$ we get

$$
\mathrm{T}_{\mathrm{m}} \Phi_{k} \mathrm{~T} e^{\Phi} \mathrm{m} \cdot \xi=\mathrm{T}_{e} \Phi_{\mathrm{m}}\left(A d_{k} \xi\right)
$$

which tells us that $\mathrm{T}_{\mathrm{m}} \Phi_{k}$ leaves $\mathrm{T}_{\mathrm{m}} O_{\mathrm{m}}$ invariant. 
Since $G_{m}$ is compact, there is a $G_{m}$ invariant inner product on $T_{m} M$. So $\left(\mathrm{T}_{\mathrm{m}} O_{\mathrm{m}}\right)^{\perp}$ is a $\mathrm{G}_{\mathrm{m}}$ invariant subspace. Using the local linearizing diffeomorphism $\psi$ (from the Bochner Lemma) the submanifold

$$
\mathrm{S}_{\varepsilon}=\psi^{-1}\left(\left(\mathrm{~T}_{\mathrm{m}} O_{\mathrm{m}}\right)^{1} \cap \mathrm{B}_{\varepsilon}\right)
$$

where $B_{\varepsilon}$ is a ball of radius $\varepsilon$ in $T_{m} M$ (with respect to the $G_{m}$ invariant inner product) is $\mathrm{G}_{\mathrm{m}}$ invariant. So $\mathrm{S}_{\varepsilon}$ has property (3). Moreover, $\mathrm{T}_{\mathrm{m}} \mathrm{S}_{\varepsilon}=\left(\mathrm{T}_{\mathrm{m}} O_{\mathrm{m}}\right)^{\perp}$, since $\mathrm{T}_{\mathrm{m}} \psi=$ identity. Hence, property (1) holds as well.

We argue that Property (2) is an open condition in $S_{\varepsilon}$ as follows. Observe that $\Phi \mid\left(\mathrm{G} \times \mathrm{S}_{\varepsilon}\right): \mathrm{G} \times \mathrm{S}_{\mathcal{\varepsilon}} \rightarrow \mathrm{M}$ is a submersion at $(e, \mathrm{~m})$. Hence it is a submersion at $(e, \mathrm{p})$, for all $\mathrm{p}$ in a neighbourhood of $\mathrm{m}$ in $\mathrm{S}_{\varepsilon}$.

Now it remains to show that we can find $\varepsilon>0$ so that (4) holds. Suppose that it does not hold for any $\varepsilon>0$. This would imply that there is a sequence of points $\left\{m_{n}\right\}$ with $\mathrm{m}_{\mathrm{n}} \in \mathrm{S}_{1 / \mathrm{n}}$, and a sequence $g_{\mathrm{n}} \in \mathrm{G}$, such that $g_{\mathrm{n}} \notin \mathrm{G}_{\mathrm{m}}$, and $g_{\mathrm{n}} \mathrm{m}_{\mathrm{n}} \in \mathrm{S}_{1 / \mathrm{n}}^{\backslash}$. Hence, $\mathrm{m}_{\mathrm{n}} \rightarrow \mathrm{m}$ and $g_{\mathrm{n}} \mathrm{m}_{\mathrm{n}} \rightarrow \mathrm{m}$. Since the action of $\mathrm{G}$ in $\mathrm{M}$ is proper, it follows that there exists convergent subsequence of $g_{\mathrm{n}}$. Without loss of generality, we may assume that $g_{\mathrm{n}}$ $\rightarrow g$. Moreover, $g_{\mathrm{n}} \mathrm{m}_{\mathrm{n}} \rightarrow g \mathrm{~m}=\mathrm{m}$, which implies that $g \in \mathrm{G}_{\mathrm{m}}$. Hence, $g^{-1} g_{\mathrm{n}} \rightarrow e,: g \in$ $\mathrm{G}_{\mathrm{m}}$ and $g_{\mathrm{n}} \notin \mathrm{G}_{\mathrm{m}}$.

$\mathrm{G}_{\mathrm{m}}$ acts in $\mathrm{G}$ be multiplication on the left, and the orbit of this action through the identity in $G$ coincides with $G_{m}$. Applying Lemma $C .1$ to the action of $G_{m}$ in $G$, we conclude that there is a submanifold $L$ of $G$ transverse to $G_{m}$ at $e$, and an open set $\mathrm{U} \times \mathrm{V} \subseteq \mathrm{G}_{\mathrm{m}} \times \mathrm{L}$ such that the multiplication $(k, l) \mapsto k l$ is a diffeomorphism onto some open neighbourhood $\mathrm{W}$ of $e$ in $\mathrm{G}$. Thus, we may assume that $g^{-1} g_{\mathrm{n}}=k_{\mathrm{n}} l_{\mathrm{n}}$, with $k_{\mathrm{n}} \in \mathrm{G}_{\mathrm{m}}$ and $l_{\mathrm{n}} \in \mathrm{L}$. Since, $g$ and $k_{\mathrm{n}}$ are in $\mathrm{G}_{\mathrm{m}}$ and $g_{\mathrm{n}} \notin \mathrm{G}_{\mathrm{m}}$, it follows that $l_{\mathrm{n}}=$ $k_{\mathrm{n}}^{-1} g^{-1} g_{\mathrm{n}} \notin \mathrm{G}_{\mathrm{m}}$ for all $\mathrm{n}$.

We now apply Lemma $\mathrm{C} .1$ to $\mathrm{U} \times \mathrm{V} \subseteq \mathrm{L} \times \mathrm{S}_{\varepsilon}$. For sufficiently large $\mathrm{n}, g_{\mathrm{n}} \mathrm{m}_{\mathrm{n}}=$ $g k_{\mathrm{n}} l_{\mathrm{n}} \mathrm{m}_{\mathrm{n}}$ is in $\mathrm{V} \subseteq \mathrm{S}_{\mathcal{E}}^{\prime}$. It follows from Corollary C.2 that $g k_{\mathrm{n}} l_{\mathrm{n}}=e$ for $\mathrm{n}$ 
large enough. Hence, $l_{\mathrm{n}}=k_{\mathrm{n}}^{-1} g^{-1} \in \mathrm{G}_{\mathrm{m}}$, which contradicts the result above. This contradiction establishes (4).

Q.E.D.

We should remark that, for the case under consideration in this paper, that is for $\mathrm{G}=G S(\mathbf{P})$, there is a natural $G S(\mathbf{P})$ invariant weak inner product on on the manifold $\mathrm{M}$ $=\mathrm{P}$ given by the $\mathrm{L}^{2}$ scalar product. In this case, we can take $\left(\mathrm{T}_{\mathrm{m}} O_{\mathrm{m}}\right)^{\perp}$ to be the $\mathrm{L}^{2}$ orthogonal complement of $\mathrm{T}_{\mathrm{m}} O_{\mathrm{m}}$. As long as, the ball $\mathrm{B}_{\varepsilon}$ is defined with respect to the stróng $\mathrm{G}_{\mathrm{m}}$ invariant inner product on $\mathrm{M}$, the manifold $\mathrm{S}_{\varepsilon}$ defined by (C.13) will satisfy properties (1) through (4). Hence, for the gauge symmetry group $G S(P)$ one can always choose a slice $S$ through $m$ satisfying the condition (3.8), requiring that $T_{m} S$ is the $L^{2}$ orthogonal complement of $\mathrm{T}_{\mathrm{m}} \mathrm{O}_{\mathrm{m}}$.

\section{References.}

[1] G. Schwarz and J. Śniatycki, "Yang-Mills and Dirac fields in a bag, existence and uniqueness theorems", Comm. Math. Phys. (to appear)

[2] J. Arms, "The structure of the solution set for the Yang-Mills equations", Math. Proc. Camb. Phil. Soc.,90 (1981), 361-372.

[3] J. Arms, J.E. Marsden and V. Moncrief, Symmetry and bifurcation of momentum maps", Comm. Math. Phys., 78 (1981), 455-478.

[4] P. Mitter and C. Viallet, "On the bundle of connections and the gauge orbit manifold in. Yang-Mills theory", Comm. Math. Phys., 79 (1981), 457 - 472.

[5] M. Atiyah and R. Boot, "The 'Yang-Mills equations over Riemann surfaces", Phil. Trans. R. Soc. London, A 308 (1982) 523-615,

[6] W. Kondracki and J. Rogulski, "On the stratification of the orbit space for the action of automorphisms on connections", Dissertationes Mathematicae, CCL, Warsaw, 1986.

[7] J. Huebschmann, "The singularities of Yang-Mills connections over a surface. I. The local model", "preprint, February 1992.

[8] J. Huebschmann, "The singularities of Yang-Mills connections over a surface. II. The stratification", preprint, February 1992. 
[9] R. Sjamaar, "Singular orbit spaces in Riemannian and Symplectic geometry", Thesis, University of Utrecht, 1990.

[10] R. Sjamaar and E. Lerman, "Stratified symplectic spaces and reduction", Ann. of Math. 134 (1991), 375-422.

[11] C.B. Morrey, Multiple Integrals in the Calculus of Variations, Springer Verlag, Berlin, 1966.

[12] T. Kato, Perturbation Theory for Linear Operators, Springer Verlag, Berlin, 1966.

[13]. A. Pietsch, Nukleare Lokalkonvexe Raüme, Akademie-Verlag, Berlin, 1965.

[14] R. Palais, "On the existence of slices for actions of noncompact Lie groups, Ann. Math., 73 (1961), 295-323.

[15] R. Cushman and L. Bates, Global Aspects of Classical Integrable Systems, in preparation.

[16] S. Bochner, "Compact groups of differentiable transformations", Ann. of Math. 46 (1945) 372-381. 